\title{
ANÁlisis AEROPALINOLÓGICO EN TRES ÁREAS DE VEGETACIÓN DENTRO del Parque Nacional El Palmar (Colón, Entre Ríos) y su RELACIÓN CON LA VEGETACIÓN LOCAL Y REGIONAL
}

\author{
NADIA E. MUÑOZ1 ${ }^{1}$ MERCEDES DI PASQUO ${ }^{1}$, FERNANDO BIGANZOLI $^{2}$ y \\ WILLIAM B. BATISTA ${ }^{2,3}$
}

\begin{abstract}
Summary: Aeropalinological analysis in three vegetation areas within El Palmar National Park (Colón, Entre Ríos) and its relationship with the local and regional vegetation. The diversity of pollen rain monthly collected during two years (2011-2013) from the atmosphere in Tauber traps located at three sites in El Palmar National Park (Entre Ríos Province) is used to characterize the source vegetation. Site 1 is a mixed area composed of grassland, palm savanna, and wetland communities, site 2 is a grassland area and site 3 is a dense palm savanna. A total of 71 pollen-grain types grouped in 43 families coming from local, regional and extra- regional areas are identified. Of them, sixteen pollen types with more than $1 \%$ of Annual Pollen Influx in at least two samples were used in this analysis. Different factors involved in quali-quantitave changes of taxa during the observation interval (e.g. pollination affinity, origin of pollen grains, canopy effect, meteorological variables) are further considered. The floral composition of each site compared to their palynoassemblages revealed that site 2 is characterized by a high abundance of Asteraeceae-Asteroideae, with an increase in the value of Vernonia (Asteraceae Cichoroidea) and Lamiaceae during the second year. Palynological composition in Sites 1 (mixed vegetation) and 3 (dense palm savanna) was dominated by Asteraeceae-Asteroideae, Myrtaceae, Blepharocalyx salicifolius, and Poaceae, with percentage fluctuations between years. Site 3 is differentiated from the other two ones by the record of Butia yatay in low frequency that showed similar percentages in both years, whereas Adiantopsis chlorophylla and Microgramma mortoniana increased during the second year.
\end{abstract}

Key words: Airborne pollen diversity, vegetation, El Palmar National Park, Entre Ríos, Argentina.

\begin{abstract}
Resumen: La diversidad de polen atmosférico colectado mensualmente durante dos años (2011-2013) en trampas Tauber en tres sitios del Parque Nacional El Palmar (provincia de Entre Ríos) es usada para caracterizar la vegetación. El sitio 1 es un área mixta compuesta por pastizales, palmares y humedales. El sitio 2 es un área de pastizales y el sitio 3 un palmar denso. Se identificaron 71 tipos polínicos comprendidos en 43 familias procedentes de fuentes locales, regionales y extraregionales. Dieciséis tipos resultaron abundantes con más de $1 \%$ del Annual Pollen Influx en al menos dos muestras. Diferentes factores relacionados con los cambios cuali-cuatitativos de los taxa en el intervalo de estudio son considerados. Por lo tanto, la composición florística de cada sitio en comparación con sus asociaciones polínicas reveló que el sitio 2 se caracteriza por una alta abundancia de Asteraeceae-Asteroideae, con un aumento en el valor de Vernonia (Asteraceae Cichoroidea) y Lamiaceae durante el segundo año. Por otro lado, una composición palinológica similar (Asteraeceae-Asteroideae, Myrtaceae, Blepharocalyx salicifolius, Poaceae) se encuentra en los sitios 1 y 3 , con tasas de variación entre ambos años. El sitio 3 se diferencia de los otros dos por el registro de Butia yatay en baja frecuencia en porcentajes similares en ambos años, en tanto que Adiantopsis chlorophylla y Microgramma mortoniana aumentaron durante el segundo año.
\end{abstract}

Palabras clave: Diversidad polínica aérea, vegetación, Parque Nacional El Palmar, Entre Ríos, Argentina.

\footnotetext{
${ }^{1}$ Laboratorio de Palinoestratigrafía y Paleobotánica, CICYTTP-CONICET, Dr. Matteri y España S/N, Diamante (E3105BWA), Entre Ríos, Argentina.

E-mail: nadiamunoz@cicyttp.org.ar, medipa@cicyttp.org.ar

2 Departamento de Métodos Cuantitativos y Sistemas de Información, Facultad de Agronomía, Universidad de Buenos Aires. Avenida San Martín 4453, 1417 Buenos Aires, Argentina.

${ }^{3}$ IFEVA, Facultad de Agronomía, Universidad de Buenos Aires. Avenida San Martín 4453, 1417 Buenos Aires, Argentina.
} 


\section{INTRODUCCIÓN}

Entre las metodologías comúnmente utilizadas en estudios palinológicos se encuentra el uso de las denominadas trampas Tauber (Tauber, 1974), las cuales colectan polen por gravedad. Estas trampas, además de colectar los distintos granos de polen que pueden encontrarse en suspensión, permiten, dada su estructura, obtener índices (i.e., Pollen Influx) utilizados en la comparación de espectros polínicos provenientes de distintas áreas de estudio. Si bien se han realizado estudios que utilizan trampas Tauber en diferentes ambientes de la República Argentina (Cuadrado, 1979; Borromei \& Quattrocchio, 1990; Majas \& Romero, 1992; Naab, 1999; Fontana et al., 2001; Fontana, 2003, 2004; Madanes \& Millones, 2004; Pérez et al., 2009), en la Mesopotamia argentina, y más precisamente en la provincia de Entre Ríos, los estudios polínicos utilizando trampas Tauber se han centrado en el Parque Nacional Pre Delta (PNPD), y en la ciudad de Diamante, ubicados en el centro-oeste de la provincia (Latorre \& Caccavari, 2006, 2010; Sanchez \& Latorre, 2011).

Además del mencionado PNPD, en la provincia de Entre Ríos se encuentra otra área protegida de importancia, el Parque Nacional El Palmar (PNEP). Este parque está ubicado en el centro-este de la provincia de Entre Ríos (Departamento de Colón), sobre la margen derecha del río Uruguay $\left(31^{\circ} 50^{\prime} \mathrm{S}-58^{\circ} 17^{\prime} \mathrm{O}\right)$ y abarca una superficie de 8500 hectáreas. Fue creado en el año 1965 con el propósito de conservar la porción de la sabana húmeda mesopotámica que contiene los palmares de Butia yatay más extensos y densos que existen (Martínez-Crovetto \& Piccinini, 1950; Dimitri \& Rial, 1955). Su finalidad se extiende, además, a la protección de otros ambientes de relevancia como patrimonio natural, como la selva en galería, los pajonales, las estepas psamófilas y los eriales (Batista et al., 2014). Los palmares de yatay de esta región conforman las sabanas más australes de América del Sur, han sido considerados como comunidades relictuales de una flora de ambiente más cálido que el actualmente (Martínez-Crovetto \& Piccinini, 1950; Soriano et al., 1991; Iriondo \& Kröhling, 2008). Un primer trabajo utilizando las trampas Tauber aquí analizadas documentó la diversidad de hongos microscópicos en cada sitio y su vinculación con la flora y con las variables climáticas (Nuñez Otaño et al., 2015).
El objetivo de este trabajo es proporcionar información sobre la dispersión del polen actual en tres áreas de diferente vegetación dentro del Parque Nacional El Palmar y establecer su relación con la vegetación local y regional.

\section{Área de estudio}

Desde el punto de vista fitogeográfico, el PNEP se encuentra en la subregión de los Campos, dentro de la región de los pastizales del Río de la Plata (Soriano et al., 1991; Royo Pallarés et al., 2005; Overbeck et al., 2007, Figura 1). La vegetación de los Campos se distingue de los pastizales Pampeanos por su mayor gran abundancia de poáceas subtropicales, principalmente de los géneros Axonopus, Paspalum, Digitaria, Schizachyrium y Bothriochloa (Burkart, 1975; Soriano et al., 1991).

El Parque Nacional El Palmar protege un ecosistema de sabana templado húmeda desarrollado sobre un paisaje ondulado de modelado fluvial ocupado por un mosaico de comunidades vegetales con diferente estructura de la vegetación (Batista et al., 2014; Ciccero \& Balabusic, 1994; Ruiz Selmo et al., 2007; Rolhauser, 2002, 2007). Las principales unidades que componen este mosaico son:

Palmar denso. Stands (áreas dentro de las cuales

la vegetación presenta estructura y composición florística homogéneas) con fisonomía de sabana densa o bosque de palmeras con estrato arbóreo superior formado exclusivamente por ejemplares adultos de Butia yatay, estratos arbóreo inferior y arbustivo discontinuos con Sebastiana commersoniana (Euphorbiaceae), Allophylus edulis (Sapindaceae) y Guettarda uruguensis (Rubiaceae), Hexachlamis edulis (Myrtaceae), Baccharis dracunculifolia (Asteraceae) e individuos altos de Andropogon lateralis (Poaceae). El estrato herbáceo es continuo, muy diverso y dominado por el Adiantopsis chlorophylla (Pteridaceae) y Bromus auleticus (Poaceae).

Arbustal con palmeras. Sabana arbustiva o pastizal con pocas palmeras altas o con palmeras juveniles, con estrato arbustivo conspicuo dominado por Baccharis dracunculifolia, B. pingraea y Acanthostyles buniifolius (Asteraceae) y estrato herbáceo con fisonomía similar al del palmar denso.

Erial. Estepa graminosa arbustiva con escasa cobertura asociada con afloramientos de arenisca 


\section{N. E. Muñoz et al. - Análisis aeropalinológico del Parque Nacional El Palmar}

o canteras de rodados abandonadas. Presenta muy escasas palmeras bajas, arbustos como Baccharis dracunculifolia, B. articulata y B. rufescens y estrato herbáceo dominado por Trachypogon spicatus y Schizachyrium microstachyum (Poaceae) y Croton laureltyanus (Euphorbiaceae).

Estepas psamófilas. Stands con fisonomía de estepa graminosa asociados con depósitos de arena masiva que afloran en diferentes partes del paisaje. A excepción de algunas palmeras aislada o de algún arbusto ocasional, carecen de estrato arbóreo así como de estrato arbustivo. El estrato herbáceo está dominado por Schizachysium microstachyum, Bromus auléticus, Moninna cuneata (Polygalaceae) y Croton laureltyanus.

Pajonal. Stands de pastizal alto asociados con vías de escurrimiento sin cauce definido. Ocasionalmente presentan palmeras aisladas sobre un estrato herbáceo muy alto dominado por Andropogon lateralis, Panicum glabripes, Paspalum quadrifarium (Poaceae) y Baccharis spicata (Asteraceae).

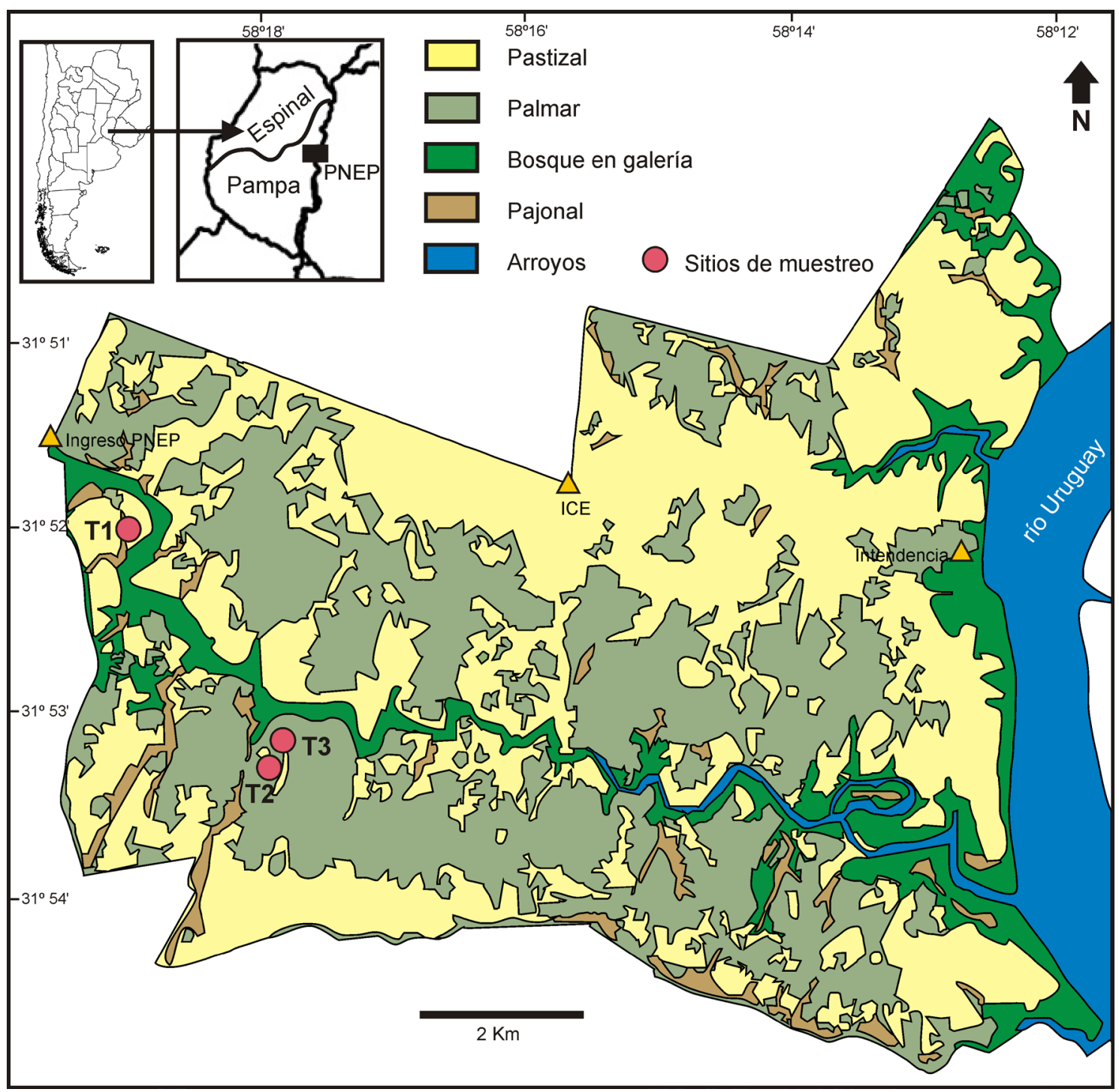

Fig. 1. Mapa del Parque Nacional El Palmar. T1-T3, sitios de muestreo. 
Bosque ripario. Stands de vegetación boscosa ubicados en las planicies aluviales del río Uruguay y de los arroyos que atraviesan el paisaje. Entre las especies más frecuentes aparecen, Pouteria salicifolia (Sapotaceae), Myrcianthes cisplatensis, M. pungens, Blepharocalyx salicifolius, Myrcia selloi, Eugenia uruguayensis (Myrtaceae), Sebastiania commersoniana, S. brasiliensis (Euphorbiaceae), Allophylus edulis (Sapindaceae), Salix humboldtiana (Salicaceae) y Ruprechtia laxiflora (Poloygonaceae).

El clima de la región en la que está ubicado el PNEP es templado húmedo, caracterizado por la influencia de los vientos húmedos del noreste, otros refrigerantes y secos del suroeste y vientos del sureste. La temperatura media anual es de $19^{\circ}$ $\mathrm{C}$ y varía entre $24,8^{\circ} \mathrm{C}$ en enero y $11,7^{\circ} \mathrm{C}$ en junio, con una amplitud de temperatura de $13,1^{\circ} \mathrm{C}$. Las heladas extremas pueden producirse desde mayo a septiembre; en tanto que la precipitación media anual es de $1346 \mathrm{~mm}$ (Rolhauser et al., 2011), siendo más abundantes en primavera / verano. Las altas temperaturas en verano inducen un frecuente déficit hídrico y aumentan las probabilidades de los eventos de incendios naturales durante este período (Goveto, 2005).

\section{Materiales y Métodos}

\section{Trabajo de campo}

Para realizar este estudio palinológico se seleccionaron tres sitios dentro de la zona sur del PNEP separada por el arroyo El Palmar, la cual no se encuentra abierta al público (Figura 1). Se instalaron tres trampas tipo Tauber colocadas a 1,50 metros de altura. Cada trampa consiste en un recipiente de $900 \mathrm{~cm}^{3}$, cubierto en su parte superior por una tapa especial que presenta un orificio central de $5 \mathrm{~cm}^{2}$, la cual permite atrapar las partículas que están suspendidas en el aire por método gravitacional. En el interior del recipiente se colocó glicerina con fenol en cantidad suficiente para cubrir la base asegurando así que todo el material depositado se conserve en condiciones adecuadas. La trampa número 1 (Tauber $1, \mathrm{~S} 31^{\circ}$ 52' 10.96"; O 58 18' 56.98", Figura 2 a) fue ubicada en un stand de estepa psamófila ubicado en la parte baja del paisaje vecino a un pajonal y a $500 \mathrm{~m}$ del bosque ripario, la trampa número 2 (Tauber 2, S $31^{\circ} 53^{\prime} 37^{\prime \prime}$; O $58^{\circ} 17^{\prime} 51$ ', Figura 2 b) en un stand de estepa psamófila a $800 \mathrm{~m}$ de un palmar denso, y la trampa número 3 (Tauber $3, \mathrm{~S}$ $31^{\circ}$ 53' 33.60"; O 58 $18^{\circ}$ ' 44.9', Figura 2 c) en el interior de un stand de palmar denso. Las trampas se recambiaron mensualmente durante dos años (marzo 2011-febrero 2013) totalizando 24 muestras por sitio. El tiempo de exposición mensual de cada trampa fue de un rango de entre 21 a 32 días. En los dos años de muestreo faltó un solo dato correspondiente a la trampa Tauber 1 del mes de marzo de 2012 debido a que cazadores furtivos volcaron la trampa interrumpiendo por unos días el proceso de colecta.

El trabajo de campo se completó con la realización de dos censos de vegetación en diciembre de 2011 y en marzo de 2012 en cada uno de los tres sitios de muestreo, donde se delimitó una parcela de $25 \mathrm{x}$ $25 \mathrm{~m}$, en la cual se registró la composición florística de la vegetación en cada uno de los siguientes cuatro estratos: 1) arbóreo superior ( $>8 \mathrm{~m}$ de altura), 2) arbóreo inferior (3 a $8 \mathrm{~m}), 3$ ) intermedio (1 a $3 \mathrm{~m}$ integrado por arbustos y/o pastos altos) y 4 ) herbáceo ( $<1 \mathrm{~m}$ de altura). En cada estrato, se asignó a cada especie registrada un valor de apreciación visual de abundancia-cobertura siguiendo la escala de Braun-Blanquet (Muller-Dombois \& Ellenbreg, 1974). La información obtenida a nivel de especie en dichos censos (Tabla 1) se agrupó en familias, a fin de ser comparable a la de los registros polínicos hallados en las trampas Tauber (Tabla 2).

La recolección de flores y otros ejemplares de plantas sin flores se realizó en forma manual sin producir daño innecesario al entorno o al mismo ejemplar del cual se obtuvo sólo una porción (flores u hojas o raíz). En el caso de los helechos se tomó una planta o una fronde (hoja) fértil de cada especie. Estos ejemplares fueron incorporados al Herbario Lorentz (DTE) del Laboratorio de Ecología vegetal del CICYTTP Diamante, y los preparados palinológicos fueron incorporados en la colección de Referencia bajo el acrónimo CICYTTP-R del Laboratorio de Palinoestratigrafía y Paleobotánica (LPP, di Pasquo \& Silvestri, 2014). Hasta el momento se lleva realizado el procesamiento de más de 80 flores, en su mayoría de formas herbáceas, y 12 especies de helechos colectados en las campañas realizadas entre 2011 y 2014 (di Pasquo et al., 2016). 


\section{N. E. Muñoz et al. - Análisis aeropalinológico del Parque Nacional El Palmar}
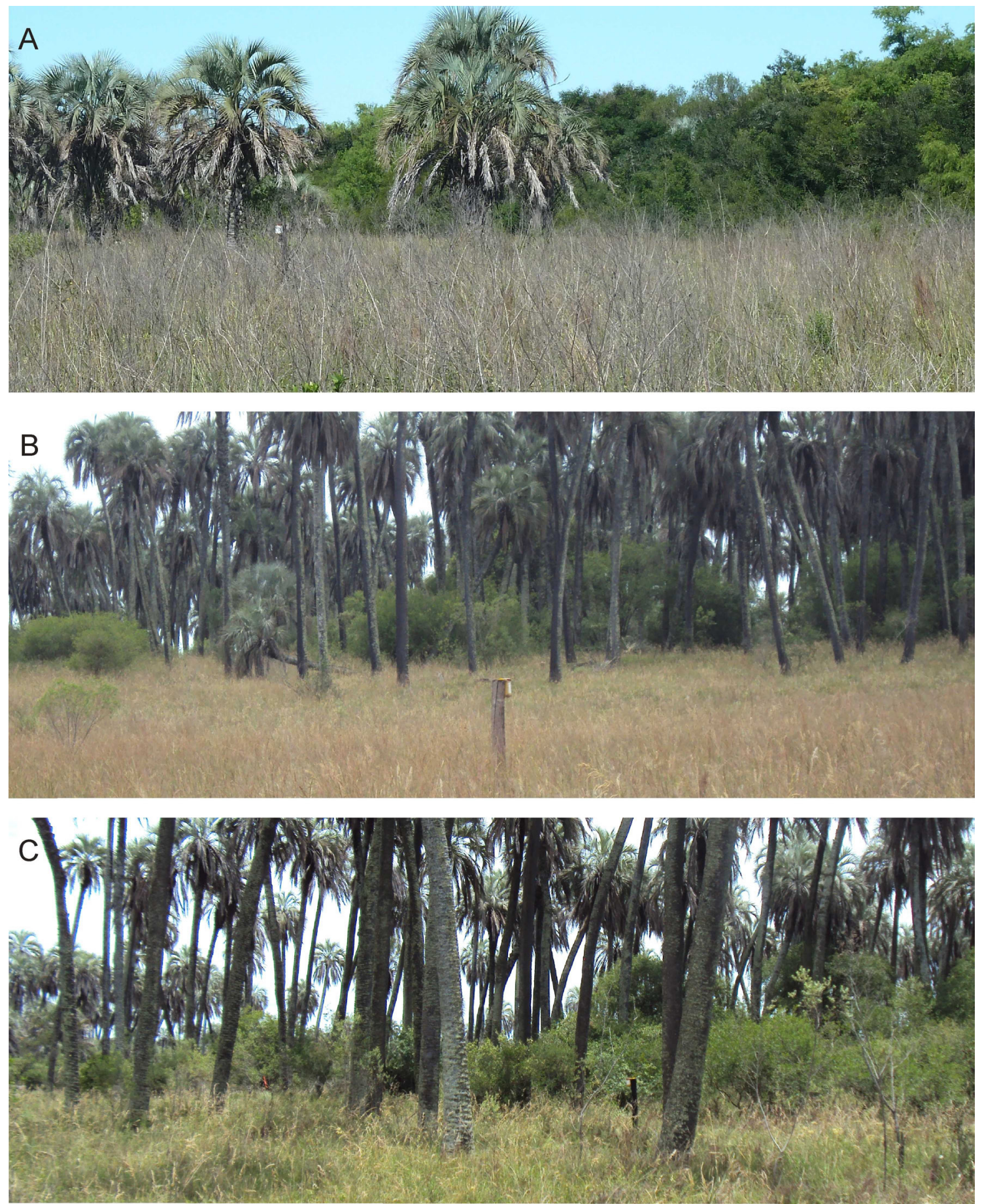

Fig. 2. A) trampa número 1 (Tauber 1) ubicada en un stand de estepa psamófila - pajonal, B) trampa número 2 (Tauber 2) en una estepa psamófila, y C) trampa número 3 (Tauber 3 ) ubicada en un Palmar denso

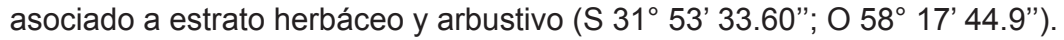


Bol. Soc. Argent. Bot. 52 (3) 2017

Tabla 1. Familias de plantas registradas en los censos de vegetación en los tres sitios Tauber.

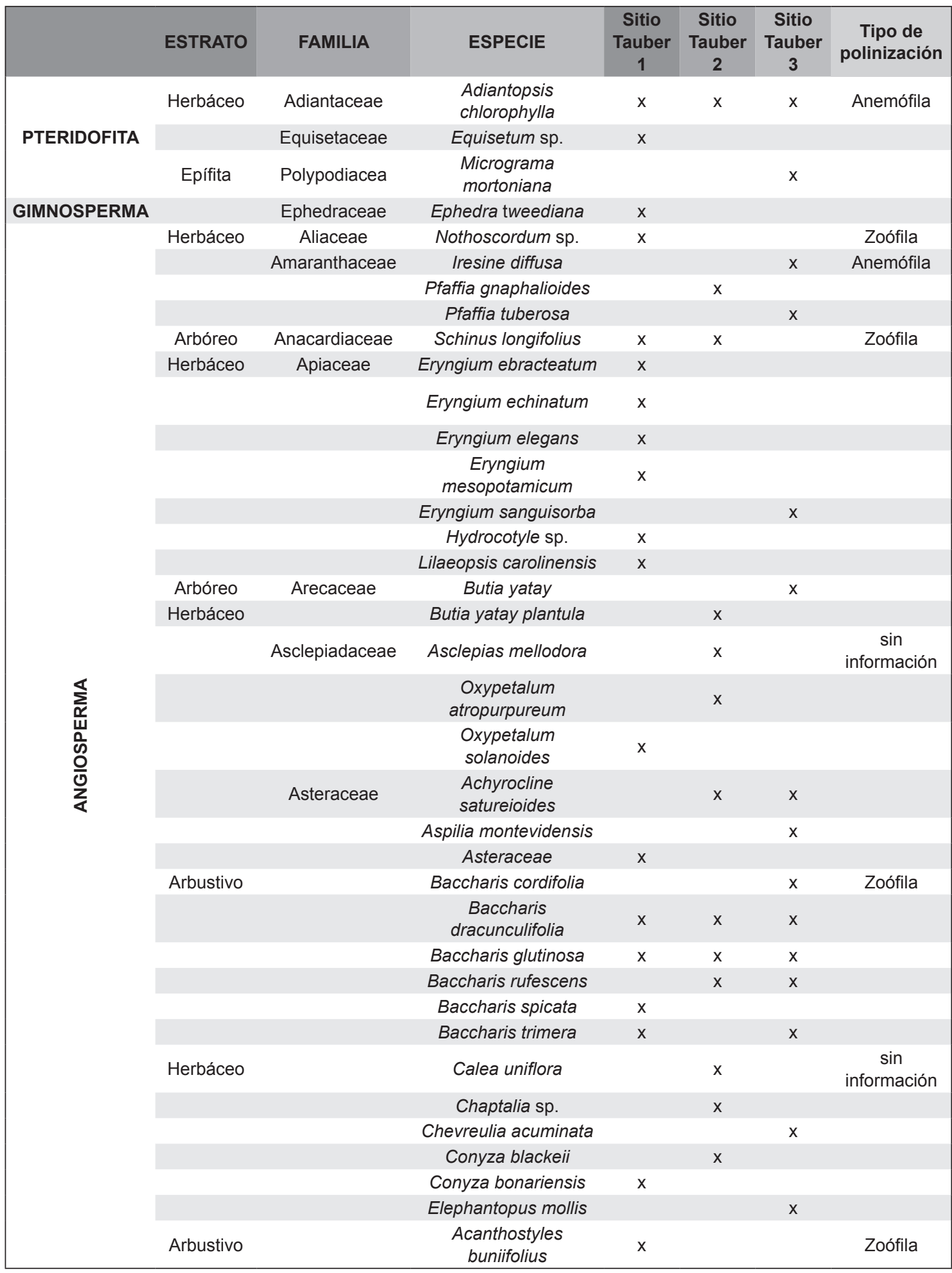


N. E. Muñoz et al. - Análisis aeropalinológico del Parque Nacional El Palmar

\begin{tabular}{|c|c|c|c|c|c|c|c|}
\hline & ESTRATO & FAMILIA & ESPECIE & $\begin{array}{c}\text { Sitio } \\
\text { Tauber } \\
1\end{array}$ & $\begin{array}{c}\text { Sitio } \\
\text { Tauber } \\
2\end{array}$ & $\begin{array}{c}\text { Sitio } \\
\text { Tauber } \\
3\end{array}$ & $\begin{array}{c}\text { Tipo de } \\
\text { polinización }\end{array}$ \\
\hline \multirow{39}{*}{ 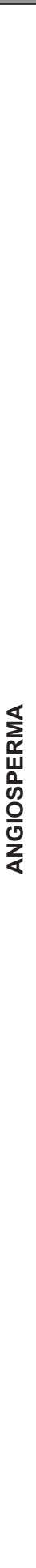 } & & & Barrosoa candelleana & & & $x$ & \\
\hline & & & $\begin{array}{l}\text { Austroepatorium } \\
\text { inulifolium }\end{array}$ & & & $x$ & \\
\hline & & & Chromolaena ivifolia & & $x$ & $x$ & \\
\hline & & & Eupathoriae sp. & $x$ & & & \\
\hline & & & Chromolaena hirsuta & & $x$ & $x$ & \\
\hline & Herbáceo & & Gamochaeta sp. & & $x$ & & $\begin{array}{c}\sin \\
\text { información }\end{array}$ \\
\hline & & & $\begin{array}{l}\text { Hieracium } \\
\text { commersonii }\end{array}$ & & & $x$ & \\
\hline & & & Jaegeria hirta & $x$ & & & \\
\hline & & & Mikania micrantha & $x$ & & & Zoófila \\
\hline & & & Mikania thapsoides & & & $x$ & \\
\hline & & & $\begin{array}{l}\text { Orthopappus } \\
\text { angustifolius }\end{array}$ & & $x$ & $\mathrm{x}$ & \\
\hline & & & Podocoma hirsuta & & & $x$ & \\
\hline & & & $\begin{array}{l}\text { Porophyllum } \\
\text { lanceolatum }\end{array}$ & & $x$ & $x$ & \\
\hline & & & $\begin{array}{l}\text { Schlechtendalia } \\
\text { luzulaefolia }\end{array}$ & & $x$ & & \\
\hline & & & Senecio grisebachii & $x$ & & & \\
\hline & & & Senecio selloi & $x$ & & & \\
\hline & & & Solidago chilensis & $x$ & & & \\
\hline & & & Stevia entreriensis & & $x$ & & \\
\hline & & & Trixis pallida & $x$ & $x$ & & \\
\hline & & & Trixis stricta & & & $x$ & \\
\hline & & & Vernonia brevifolia & & & $x$ & \\
\hline & & & Vernonia platensis & $x$ & $x$ & $x$ & \\
\hline & & & Vernonia rubricaulis & & & $x$ & \\
\hline & & & Vernonia sellowi & & & $x$ & \\
\hline & & Begoniaceae & Begonia cucullata & $x$ & & & \\
\hline & & Brassicaceae & Lepidium sp. & $x$ & & & \\
\hline & & Bromeliaceae & Dyckia ferox & & & $x$ & \\
\hline & & & Tillandsia sp. & & & $x$ & \\
\hline & & Campanulaceae & Pratia hederacea & $x$ & & & \\
\hline & & & $\begin{array}{c}\text { Wahlenbergia } \\
\text { linarioides }\end{array}$ & & $x$ & $x$ & \\
\hline & & Cariophyllaceae & Cardionema rosetta & & & & \\
\hline & Arbóreo & Celastraceae & Maytenus ilicifolia & & & $x$ & \\
\hline & Herbáceo & Cistaceae & $\begin{array}{c}\text { Crocanthemum } \\
\text { brasiliensis (Halimium } \\
\text { brasiliensis) }\end{array}$ & & & & \\
\hline & Arbóreo & Combretaceae & Terminalia australis & $x$ & & & \\
\hline & Herbáceo & Commelinaceae & Commelina erecta & & & $x$ & \\
\hline & & & $\begin{array}{l}\text { Commelina } \\
\text { plathyphylla }\end{array}$ & $\mathrm{x}$ & & & \\
\hline & & & $\begin{array}{l}\text { Tripogandra } \\
\text { glandulosa }\end{array}$ & & $\mathrm{x}$ & $x$ & \\
\hline & & Convolvulaceae & Dichondra sericea & & $x$ & $x$ & \\
\hline & & & Evolvulus sericeus & & $x$ & $x$ & \\
\hline
\end{tabular}


Bol. Soc. Argent. Bot. 52 (3) 2017

\begin{tabular}{|c|c|c|c|c|c|c|c|}
\hline & ESTRATO & FAMILIA & ESPECIE & $\begin{array}{c}\text { Sitio } \\
\text { Tauber } \\
1\end{array}$ & $\begin{array}{c}\text { Sitio } \\
\text { Tauber } \\
2 \\
\end{array}$ & $\begin{array}{c}\text { Sitio } \\
\text { Tauber } \\
3\end{array}$ & $\begin{array}{c}\text { Tipo de } \\
\text { polinización }\end{array}$ \\
\hline \multirow{44}{*}{$\begin{array}{l}\frac{\mathbb{1}}{0} \\
\frac{1}{w} \\
\frac{0}{0} \\
\frac{0}{0} \\
\frac{0}{z}\end{array}$} & & Cyperaceae & Bulbostylis capillaris & & & & Anemófila \\
\hline & & & Carex sp. & $x$ & $x$ & $x$ & \\
\hline & & & Cypepracea sp. & $x$ & & & \\
\hline & & & Cyperus reflexus & & $x$ & & \\
\hline & & & Cyperus rotundus & & & $x$ & \\
\hline & & & Cyperus sp. & & & $x$ & \\
\hline & & & Eleocharis sp. & $x$ & & & \\
\hline & & Escalloniaceae & $\begin{array}{c}\text { Escallonia } \\
\text { montevidensis }\end{array}$ & $x$ & & & $\begin{array}{c}\sin \\
\text { información }\end{array}$ \\
\hline & & Euphorbiaceae & Acalypha communis & & $x$ & $x$ & Zoófila \\
\hline & & & Bernardia sellowi & & $x$ & $x$ & \\
\hline & & & Croton glandulosus & & $x$ & & \\
\hline & & & Croton gnaphalii & $x$ & & & \\
\hline & & & Croton gnaphalioides & & $x$ & $x$ & \\
\hline & & & Euphorbia serpens & & & $x$ & \\
\hline & & & Euphorbiaceae & $x$ & & & \\
\hline & & & Tragia geraniifolia & & & $x$ & \\
\hline & Arbóreo & Fabaceae & Acacia caven & $x$ & & & \\
\hline & Herbáceo & & $\begin{array}{l}\text { Aeschynomene } \\
\text { histrix var incana }\end{array}$ & & $\mathrm{x}$ & & \\
\hline & & & Desmanthus virgatus & & & $x$ & \\
\hline & & & Desmodium incanum & $x$ & & & \\
\hline & Arbóreo & & Erythrina crista galli & $x$ & & & \\
\hline & Herbáceo & & Galactia marginalis & & & $x$ & \\
\hline & & & Indigofera asperifolia & & & $x$ & \\
\hline & & & Lupinus bracteolaris & & & $x$ & \\
\hline & & & $\begin{array}{l}\text { Rhynchosia } \\
\text { diversifolia }\end{array}$ & $x$ & & $x$ & \\
\hline & & & Rhynchosia senna & & & $x$ & \\
\hline & & & Tephrosia cinerea & $x$ & & & \\
\hline & & & Vicia pampicola & $x$ & & & \\
\hline & & & Zornia latifolia & & $x$ & & \\
\hline & & Gesneriaceae & $\begin{array}{l}\text { Corytholoma } \\
\text { alagophyllum }\end{array}$ & & & $x$ & \\
\hline & & Hypericaceae & Hypericum brasiliense & $x$ & & & \\
\hline & & Iridaceae & Gelasine azurea & $x$ & & & $\begin{array}{l}\text { Zoófila/ } \\
\text { anemófila }\end{array}$ \\
\hline & & & Sisyrinchium sp. & & $x$ & & \\
\hline & & Juncaceae & Juncaceae & $x$ & & & Anemófila \\
\hline & & & Juncus sp. & $x$ & & & \\
\hline & & Lamiaceae & Hyptis mutabilis & & $x$ & $x$ & Zoófila \\
\hline & & & Lamiaceae & $x$ & & & \\
\hline & & Lythraceae & Cuphea glutinosa & $x$ & & & \\
\hline & & & Heimia salicifolia & $x$ & & & \\
\hline & & Malpighiaceae & Janusia guaranitica & $x$ & & & \\
\hline & & Malvaceae & Cienfuegosia sulfurea & & & $x$ & \\
\hline & & & Hibiscus striatus & $x$ & & & \\
\hline & & & Pavonia hastata & $x$ & $x$ & $x$ & \\
\hline & & & Sida multicrena & & & $x$ & \\
\hline
\end{tabular}


N. E. Muñoz et al. - Análisis aeropalinológico del Parque Nacional El Palmar

\begin{tabular}{|c|c|c|c|c|c|c|c|}
\hline & ESTRATO & FAMILIA & ESPECIE & $\begin{array}{c}\text { Sitio } \\
\text { Tauber } \\
1\end{array}$ & $\begin{array}{c}\text { Sitio } \\
\text { Tauber } \\
2\end{array}$ & $\begin{array}{c}\text { Sitio } \\
\text { Tauber } \\
3\end{array}$ & $\begin{array}{c}\text { Tipo de } \\
\text { polinización }\end{array}$ \\
\hline \multirow{45}{*}{ 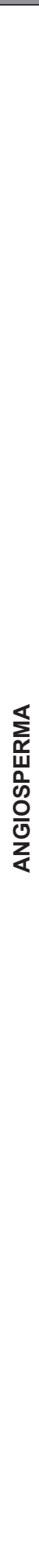 } & & & Sida rhombifolia & & & $x$ & \\
\hline & & & $\begin{array}{c}\text { Wissadula } \\
\text { glechomaefolia }\end{array}$ & & & $\mathrm{x}$ & \\
\hline & & Melastomataceae & Tibouchina gracilis & $x$ & & & \\
\hline & & Moraceae & Dorstenia brasiliensis & $\mathrm{x}$ & & & Anemófila \\
\hline & Arbóreo & Myrtaceae & Eugenia myrcianthes & & & $x$ & Zoófila \\
\hline & & & Myrcia selloi & & $x$ & $x$ & \\
\hline & & & $\begin{array}{l}\text { Myrcianthes } \\
\text { cysplatensis }\end{array}$ & & $x$ & $x$ & \\
\hline & & & Psidium incanum & $x$ & & & \\
\hline & Herbáceo & Onagraceae & Oenothera indecora & & $x$ & & \\
\hline & & & Oenothera longiflora & & $x$ & & \\
\hline & & & Oenothera sp. & $x$ & & & \\
\hline & Epífita & Orchidaceae & Orchideaceae & $x$ & & & \\
\hline & Herbáceo & Oxalidaceae & Oxalis conorrhiza & & $x$ & & \\
\hline & & & Oxalis sp. & $x$ & & $x$ & \\
\hline & & Passifloraceae & Passiflora caerulea & & $x$ & $x$ & \\
\hline & & & Passiflora chrysophylla & & $x$ & $x$ & \\
\hline & & Plantaginaceae & Angelonia integerrima & & $x$ & $x$ & \\
\hline & & & Plantago brasiliensis & & $x$ & & Anemófila \\
\hline & & Poaceae & Andropogon lateralis & $x$ & & $x$ & \\
\hline & & & Aristida sp. & & $x$ & & \\
\hline & & & Axonopus argentinus & & $x$ & & \\
\hline & & & Briza calotheca & & $x$ & & \\
\hline & & & Briza minor & $x$ & & & \\
\hline & & & Briza rufa & $x$ & & & \\
\hline & & & Briza subaristata & & $x$ & $x$ & \\
\hline & & & Briza uniolae & $x$ & & & \\
\hline & & & Bromus auleticus & & $x$ & $x$ & \\
\hline & & & Chloris retusa & & $x$ & $x$ & \\
\hline & & & Chloris sp. & $x$ & & & \\
\hline & & & $\begin{array}{c}\text { Deyeuxia } \\
\text { viridiflavescens }\end{array}$ & $x$ & & & \\
\hline & & & Digitaria swalleniana & & $x$ & $x$ & \\
\hline & & & Eragrostis airoides & & $x$ & & \\
\hline & & & Eragrostis lugens & $x$ & & & \\
\hline & & & Festuca ampliflora & & & $x$ & \\
\hline & & & Gymnopogon biflorus & & $x$ & & \\
\hline & & & $\begin{array}{l}\text { Leptocoriphium } \\
\text { lanatum }\end{array}$ & $x$ & & & \\
\hline & & & Lolium multiflorum & & & $x$ & \\
\hline & & & Melica rigida & & $x$ & $x$ & \\
\hline & & & Mnesithea selloana & & $x$ & $x$ & \\
\hline & & & Panicum grumosum & $x$ & & & \\
\hline & & & Panicum milloides & $x$ & & & \\
\hline & & & Panicum prionitis & $x$ & & & \\
\hline & & & Panicum sabulorum & $x$ & & $x$ & \\
\hline & & & Pappophorum sp. & & $x$ & $x$ & \\
\hline & & & Paspalum juerguensii & & & $x$ & \\
\hline
\end{tabular}


Bol. Soc. Argent. Bot. 52 (3) 2017

\begin{tabular}{|c|c|c|c|c|c|c|c|}
\hline & ESTRATO & FAMILIA & ESPECIE & $\begin{array}{c}\text { Sitio } \\
\text { Tauber } \\
1\end{array}$ & $\begin{array}{c}\text { Sitio } \\
\text { Tauber } \\
2\end{array}$ & $\begin{array}{c}\text { Sitio } \\
\text { Tauber } \\
3\end{array}$ & $\begin{array}{c}\text { Tipo de } \\
\text { polinización }\end{array}$ \\
\hline \multirow{36}{*}{ 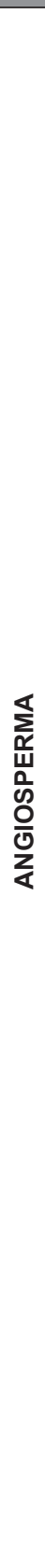 } & & & $\begin{array}{l}\text { Paspalum plicatulum } \\
\text { (o nicorae) }\end{array}$ & & $x$ & $x$ & \\
\hline & & & $\begin{array}{l}\text { Paspalum plicatulum } \\
\text { var villosissimum }\end{array}$ & & & $x$ & \\
\hline & & & Paspalum rufum & $x$ & & & \\
\hline & & & Phalaris sp. & $x$ & & & \\
\hline & & & $\begin{array}{l}\text { Piptochaetium } \\
\text { montevidensis }\end{array}$ & & $\mathrm{x}$ & $x$ & \\
\hline & & & $\begin{array}{l}\text { Piptochaetium } \\
\text { uruguensis }\end{array}$ & & $\mathrm{x}$ & & \\
\hline & & & Poa lanigera & & $x$ & & \\
\hline & & & Poa lanuginosa & & & $x$ & \\
\hline & & & Poaceae & $x$ & & & \\
\hline & & & Schizachyrium sp. & $x$ & $x$ & $x$ & \\
\hline & & & Setaria geniculata & & $x$ & $x$ & \\
\hline & & & Stipa aff brachichaeta & $x$ & & & \\
\hline & & & Stipa melanosperma & & $x$ & $x$ & \\
\hline & & & Stipa sp. & & & $x$ & \\
\hline & & & Trachipogon montufari & & & $x$ & \\
\hline & & & Tridens brasiliensis & $x$ & & & \\
\hline & & Polygalaceae & Monina cuneata & & $x$ & & Zoófila \\
\hline & & & Monnina sp. & $x$ & & $x$ & \\
\hline & & & Polygonum sp. & $x$ & & & \\
\hline & & Rubiaceae & $\begin{array}{c}\text { Galianthe } \\
\text { centranthoides }\end{array}$ & & $x$ & $x$ & \\
\hline & & & Galium richardianum & $x$ & & & \\
\hline & & & Galium sp. & $x$ & & $x$ & \\
\hline & & & Guetarda uruguensis & & & $x$ & \\
\hline & & & $\begin{array}{c}\text { Mitracarpus } \\
\text { megapotamicus }\end{array}$ & $x$ & & & \\
\hline & & & Richardia brasiliensis & & $x$ & $x$ & \\
\hline & Arbóreo & Sapindaceae & Allophylus edulis & $x$ & & $x$ & \\
\hline & Herbáceo & Scrophulariaceae & Mecardonia tenella & $x$ & & & Anemófila \\
\hline & & & $\begin{array}{c}\text { Scoparia } \\
\text { montevidensis }\end{array}$ & $x$ & & & Zoófila \\
\hline & & Smilacaceae & Smilax campestris & & & $x$ & \\
\hline & & Solanaceae & Petunia integrifolia & & $x$ & $x$ & \\
\hline & & & Petunia thymifolia & & $x$ & & \\
\hline & & & Solananum platense & $x$ & & & \\
\hline & & & Solanum commersonii & $x$ & & & \\
\hline & & Thymelaeaceae & Daphnopsis racemosa & & & $x$ & \\
\hline & & Verbenaceae & Lantana camara & & & $x$ & \\
\hline & & & Lantana $x$ entrerriensis & & & $x$ & \\
\hline
\end{tabular}

Trabajo de laboratorio

Las muestras aeropalinológicas fueron concentradas y acetolizadas durante 2 minutos, siguiendo la metodología estándar(Faegri \& Iversen, 1989). Para el cálculo posterior de frecuencia polínica Pollen Influx (Hicks, 1997; Hicks \& Hyvärinen, 1999) y previo a todo tratamiento, se incorporó una (1) tableta de un marcador foráneo compuesto por esporas de Lycopodium clavatum (Batch $N^{\circ}$ 177745) (Stockmar, 1971). En cada 
N. E. Muñoz et al. - Análisis aeropalinológico del Parque Nacional El Palmar

Tabla 2. Familias de plantas censadas en cada sitio Tauber y familias de taxones polínicos presentes en cada trampa Tauber (43 familias correspondientes a las filas sombreadas).

\begin{tabular}{|c|c|c|c|c|c|c|c|}
\hline & \multirow{2}{*}{ FAMILIA } & \multicolumn{2}{|c|}{ trampa Tauber 1} & \multicolumn{2}{|c|}{ trampa Tauber 2} & \multicolumn{2}{|c|}{ trampa Tauber 3} \\
\hline & & vegetación & polen & vegetación & polen & vegetación & polen \\
\hline \multirow{3}{*}{ PTERIDOFITAS } & Adiantaceae & $x$ & $x$ & $x$ & $x$ & $x$ & $x$ \\
\hline & Polypodiacea & & $x$ & $x$ & $x$ & $x$ & $x$ \\
\hline & Equisetaceae & $x$ & & & & & \\
\hline \multirow{2}{*}{ GIMNOSPERMA } & Ephedraceae & & $x$ & & $x$ & & $x$ \\
\hline & Pinaceae & & $x$ & & $x$ & & $x$ \\
\hline \multirow{37}{*}{ ANGIOSPERMA } & Aliaceae & $x$ & & & & & \\
\hline & Amaranthaceae & & $x$ & & $\mathrm{x}$ & $\mathrm{x}$ & $x$ \\
\hline & Anacardiaceae & $x$ & $x$ & $x$ & $x$ & $x$ & \\
\hline & Apiaceae & $\mathrm{x}$ & $x$ & $\mathrm{x}$ & & $\mathrm{x}$ & $\mathrm{x}$ \\
\hline & Arecaceae & $x$ & $x$ & & $x$ & $x$ & $x$ \\
\hline & Asclepiadaceae & & & $x$ & & & \\
\hline & Asteraceae & $x$ & $\mathrm{x}$ & $x$ & $x$ & $\mathrm{x}$ & $x$ \\
\hline & Betulaceae & & $x$ & & $\mathrm{x}$ & & $x$ \\
\hline & Boraginaceae & & $x$ & & $x$ & & $x$ \\
\hline & Brassicaceae & $x$ & $x$ & & $x$ & & $x$ \\
\hline & Bromeliaceae & & & $x$ & & $\mathrm{x}$ & $x$ \\
\hline & Campanulaceae & $x$ & $x$ & $x$ & & $x$ & \\
\hline & Cariophyllaceae & & & $x$ & $x$ & & \\
\hline & Casuarinaceae & & $\mathrm{x}$ & & $x$ & & $\mathrm{x}$ \\
\hline & Celastraceae & & $x$ & & $x$ & & \\
\hline & Celtidaceae & & $\mathrm{x}$ & & $\mathrm{x}$ & & $\mathrm{x}$ \\
\hline & Combretaceae & $x$ & & & & & \\
\hline & Cistaceae & $x$ & & & & & \\
\hline & Commelinaceae & $x$ & & & $x$ & $x$ & $x$ \\
\hline & Convolvulaceae & & $x$ & $x$ & $x$ & $x$ & $x$ \\
\hline & Cyperaceae & $x$ & $x$ & $x$ & $x$ & $x$ & $x$ \\
\hline & Escalloniaceae & $x$ & & & $x$ & & $x$ \\
\hline & Euphorbiaceae & $x$ & $x$ & $\mathrm{x}$ & $x$ & $x$ & $x$ \\
\hline & Fabaceae & $x$ & & $x$ & $x$ & $x$ & \\
\hline & Gentianaceae & & & & $x$ & & \\
\hline & Gesneriaceae & & & & & $x$ & \\
\hline & Iridaceae & $x$ & $x$ & $x$ & $x$ & & $x$ \\
\hline & Juncaceae & $x$ & & & & & \\
\hline & Lythraceae & $x$ & & & & & \\
\hline & Malpighiaceae & $x$ & & & & & \\
\hline & Lamiaceae & & $x$ & $x$ & $x$ & $x$ & $x$ \\
\hline & Malvaceae & $x$ & & $\mathrm{x}$ & & $x$ & $x$ \\
\hline & Melastomataceae & $x$ & & & & & \\
\hline & Moraceae & $x$ & & & & & \\
\hline & Myrtaceae & $x$ & $x$ & $x$ & $x$ & $x$ & $x$ \\
\hline & Nothofagaceae & & $x$ & & $\mathrm{x}$ & & $x$ \\
\hline & Oleaceae & & $\mathrm{x}$ & & $x$ & & $\mathrm{x}$ \\
\hline
\end{tabular}




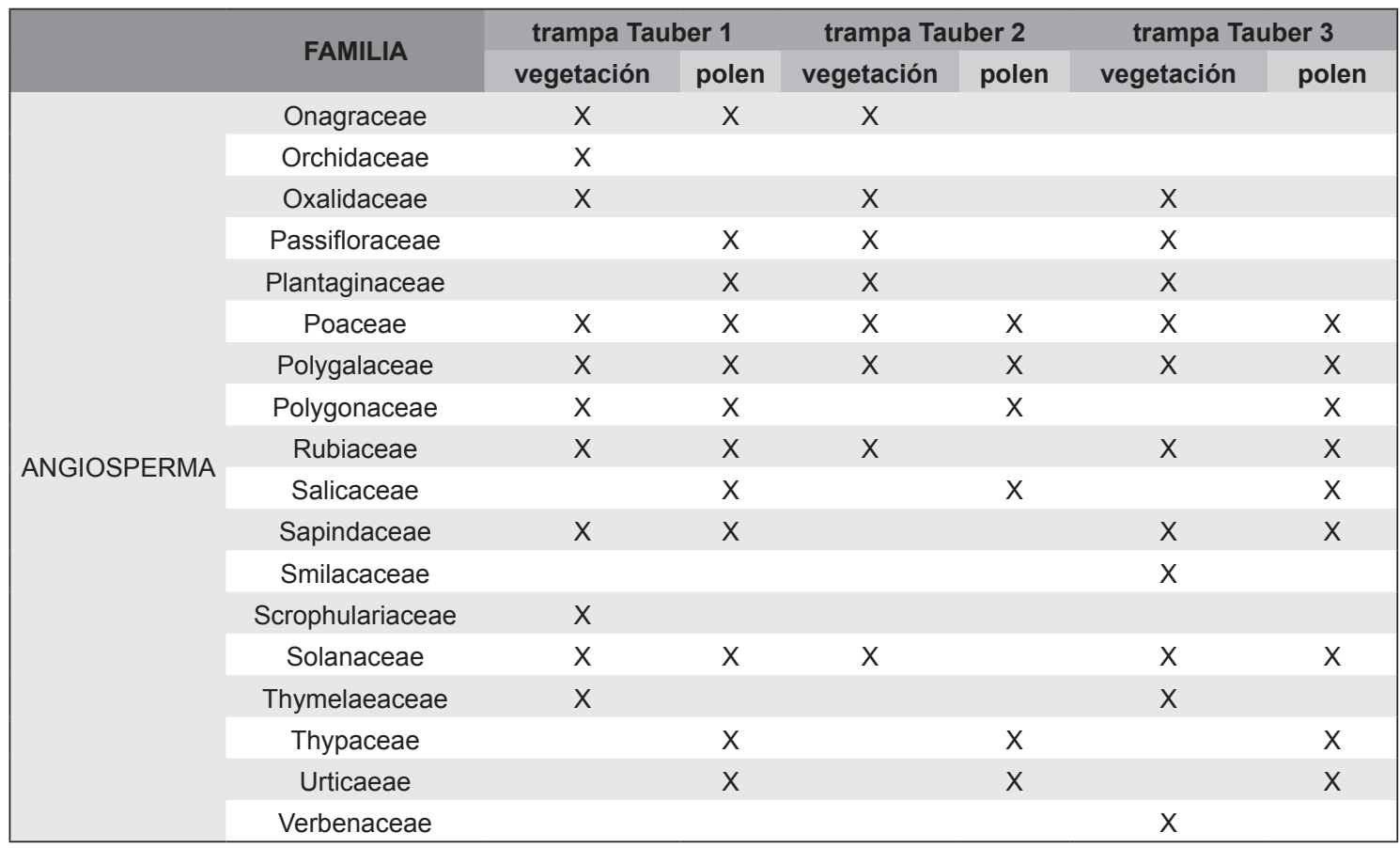

muestra se contaron un mínimo de 300 granos de polen y esporas de helechos. Sin embargo en algunas muestras de los meses de otoño e invierno el contenido de granos fue menor a 300 pero se superó el recuento de al menos 150 granos (Madanes \& Dadon, 1998).

Se calcularon los valores de abundancia polínica, expresados en número de granos por superficie de depositación $\left(\right.$ polen $/ \mathrm{cm}^{2}$ ), para los períodos mensuales y su resultado anual (Annual Pollen Influx, API). Se consideraron taxones abundantes a los tipos polínicos con al menos el $1 \%$ del total anual (\%API) en al menos dos muestras. Cabe destacar que si bien Butia yatay en una única muestra llegó al 1\% del total anual, fue considerada dentro de los taxones abundantes debido a que es el componente arbóreo predominante dentro del parque. Además, dentro las Asteraceae sólo se diferenciaron los géneros Vernonia, Mutisia y tipo Ambrosia debido a que las características morfológicas de los granos de esta familia son muy complejas y similares entre sí. Para el caso de las Myrtaceae se separó a Eucalyptus sp. por ser la única Myrtaceae exótica. La vegetación regional está representada por taxones exóticos presentes en la vegetación de sitios cercanos al parque
(Alnus sp., Casuarina cunninghamiana) y de los alrededores (Pinus sp., Eucalyptus sp.) como de Nothofagus sp. (extraregional).

Se realizaron varios ensayos estadísticos utilizando el programa PAST (Hammer et al., 2001), en los cuales se procesaron los datos de muestras aeropalinológicas, y censos de vegetación con el fin de analizar la correspondencia entre la vegetación y la composición cualitativa y cuantitativa de las asociaciones palinológicas de cada sitio Tauber.

La identificación taxonómica de los tipos polínicos se realizó a partir del material colectado en el parque (flores y helechos) entre 2011 y 2013, el cual forma parte de las colecciones de referencia mencionadas más arriba. Asimismo, se consultó el material de referencia del laboratorio de Actuopalinología del CICYTTP y bibliografía específica (e.g., Heusser, 1971; Markgraf \& D'Antoni, 1978; Roubik \& Moreno, 1991; Pire et al., 1998, 2001, 2006; entre otros). La nomenclatura de los taxones sigue a Zuloaga et al. (2008). Los palinomorfos y en particular los granos de polen presentes en los preparados palinológicos fueron estudiados bajo microscopio óptico marca Leica DM 500 e ilustrados con su videocámara Leica EC3 (3 Megapixels). Este microscopio cuenta 


\section{N. E. Muñoz et al. - Análisis aeropalinológico del Parque Nacional El Palmar}

además con un sistema de fluorescencia (filtro para fluoresceína), el cual fue utilizado para mejorar la observación de la morfología de los granos complejos.

Los datos meteorológicos de temperatura $\left({ }^{\circ} \mathrm{C}\right)$ y pluviosidad $(\mathrm{mm})$ utilizados en este estudio, son los registrados durante los dos años de muestreo por la estación meteorológica (ICE) ubicada dentro del parque (Figura 1). Asumimos que dichos datos meteorológicos son los mismos en los tres sitios estudiados debido a su proximidad.

\section{Resultados}

\section{Muestreo polínico}

El muestreo de polen atmosférico depositado en tres ambientes diferentes (Figs. 1, 2) del PNEP entre marzo 2011 y febrero 2013 arrojó valores máximos en los meses más cálidos (septiembre a febrero), mientras fue más bajo en los meses más fríos (junio a agosto).

El monitoreo anual de la Tauber 1 arrojó un API de 14876 polen/ $\mathrm{cm}^{2}$ durante el primer año (marzo 2011-febrero 2012) y de $29147 \mathrm{polen} / \mathrm{cm}^{2}$ en el segundo año (abril 2012-febrero 2013); para la Tauber 2 el API fue 49032polen $/ \mathrm{cm}^{2}$ en el primer año y de 52944 polen $/ \mathrm{cm}^{2}$ durante el segundo año; en tanto que para la Tauber 3 los valores de API fueron 39001 polen/ $/ \mathrm{cm}^{2}$ y de 39003 polen/ $\mathrm{cm}^{2}$ respectivamente. La trampa Tauber 1 (Figura 3a) presentó tres picos elevados de Pollen Influx durante febrero y septiembre de 2012 y febrero de 2013; la trampa Tauber 2 (Figura 3 b) tuvo sus máximos durante marzo de 2011 y octubre de 2012; y la trampa Tauber 3 (Figura 3 c) durante el intervalo diciembre de 2011-febrero de 2012 y durante el intervalo octubre de 2012-febrero de 2013.

Los tres sitios se caracterizaron por la presencia de Asteraceae-Asteroideae y Poaceae como elementos más comunes. Se determinaron 71 tipos polínicos pertenecientes a 43 familias incluidas en la Tabla 2. Del total de tipos polínicos, 16 resultaron abundantes (al menos 1\% de API, Tabla 3, Figura 4, A-P): Butia yatay, Celtis sp. (Celtidaceae), tipo Ambrosia (Asteraceae), Vernonia sp. (Asteraceae), Sisyrinchium sp. (Iridiaceae), Hidrocotyle sp. (Apiaceae), Myrtaceae, Blepharocalyx salicifolius (Myrtaceae), Lamiaceae, Poaceae, Cyperaceae,
Brassicaceae, Urticaeae, Asteraeceae-Asteroideae, y las esporas de helechos Adiantopsis chlorophylla (Pteridaceae) y Microgramma mortoniana (Polypodiaceae). Además, se registraron taxones exóticos como Pinus sp., Eucalyptus sp, Alnus sp., Casuarina cunninghamiana y Nothofagus sp. (Figura 4, Q-T). El $72 \%$ de los taxones hallados en las trampas Tauber corresponden a plantas que poseen polinización zoófila y el $28 \%$ anemófila (Silva et al., 2014; Tabla 1).

Sin embargo, los valores de API de los taxones analizados permiten diferenciar las tres áreas de estudio que se caracterizan de la siguiente manera (Tabla 3):

TAUBER 1. Marcada presencia de AsteraceaeAsteroideae, Myrtaceae, Poaceae y Blepharocalyx salicifolius. Otros taxones como Hidrocotyle sp., Cyperaceae y Urticaceae presentaron valores altos de API durante el primer año; en tanto que Poaceae, Brassicaceae, Blepharocalyx salicifolius y el tipo Ambrosia revelaron valores más altos en el segundo año. Butia yatay se encuentra pobremente representada en ambos años.

TAUBER 2. Predominio de AsteraceaeAsteroideae, y presencia de Myrtaceae, Poaceae y Sisyrinchium sp. (Tabla 3). AsteraceaeAsteroideae, Vernonia sp. y Lamiaceae presentaron los mayores valores tanto en el primer año como en el segundo. El tipo Ambrosia se encontró con valores altos durante el primer año y Butia yatay, escasamente representada.

TAUBER 3. Poaceae y Asteraceae-Asteroideae presentan alto valor API seguidas de Sisyrinchium sp. y Myrtaceae (Tabla 3). Poaceae, Urticaceae, Microgramma mortoniana, Adiantopsis chlorophylla y Butia yatay presentan los mayores valores en los dos años de muestreo.

Censos de vegetación y su relación con el polen Se reconocieron a nivel de género y especie 201 plantas agrupadas en 50 familias en las tres zonas de estudio (Tabla 1). En la zona de la trampa Tauber 1 se reconocieron 36-familias de plantas entre las que dominaron Poaceae, Carex sp. (Cyperaceae), Juncaceae y Janusia guaranitica (Malpighiaceae); de ellos el registro polínico representó 45,2 \% de las familias. En la zona de la trampa Tauber 2 se reconocieron 24 familias de plantas, y el registro polínico reflejó el 48,3 \% de los 
Bol. Soc. Argent. Bot. 52 (3) 2017
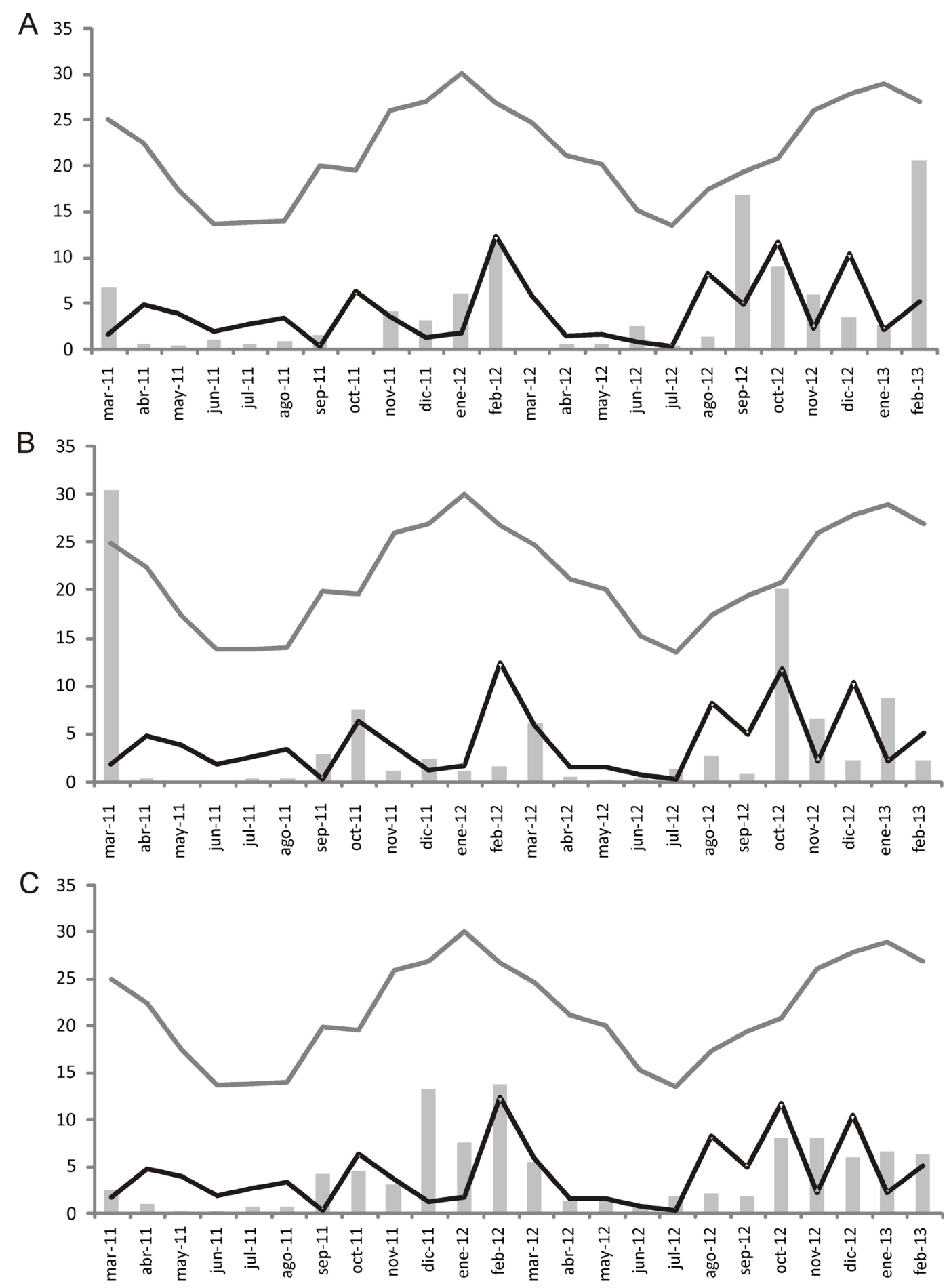

Pollen Influx $\wedge$ Temperatura $\left({ }^{\circ} \mathrm{C}\right) \wedge$ Precipitación $(\mathrm{mm})$

Fig. 3. Polen influx mensual y variables meteorológicas. A) Trampa Tauber 1; B) Trampa Tauber 2; C) Trampa Tauber 3. 


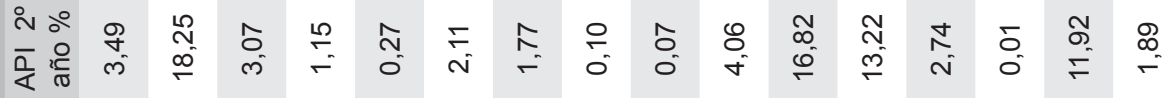

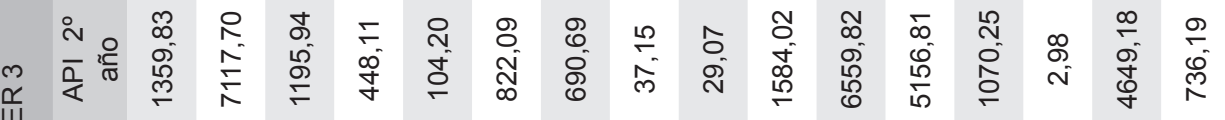

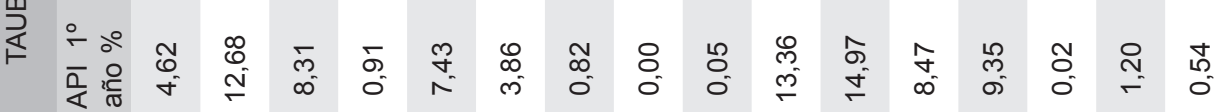

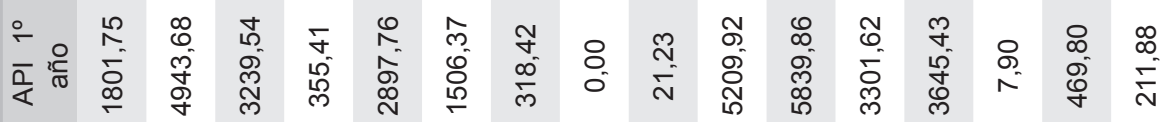

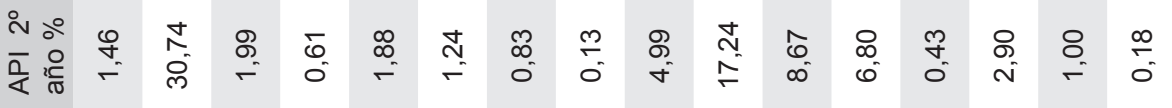

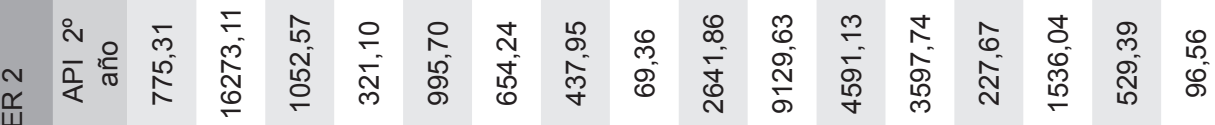
焉

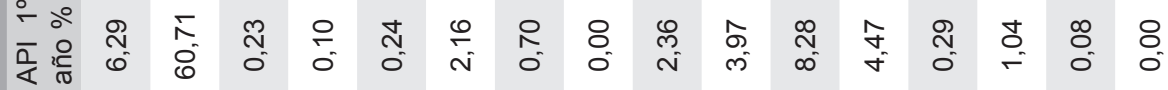

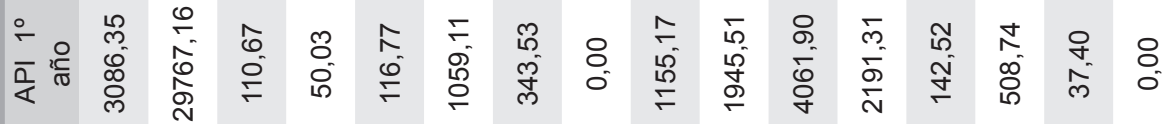

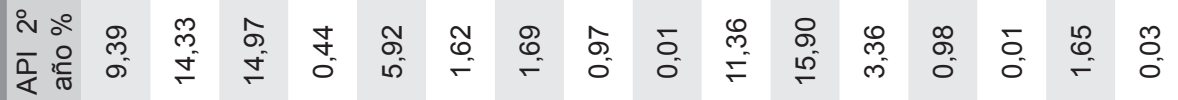

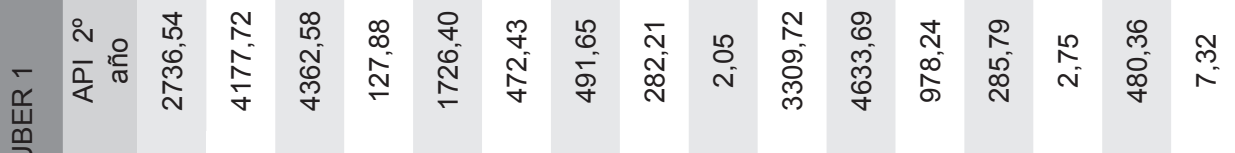

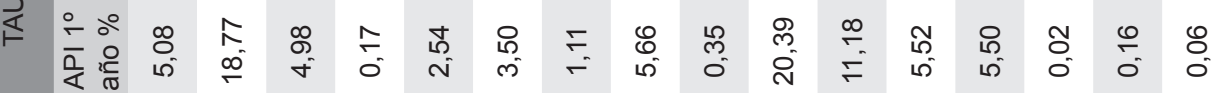

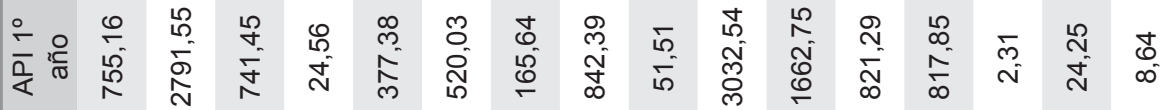

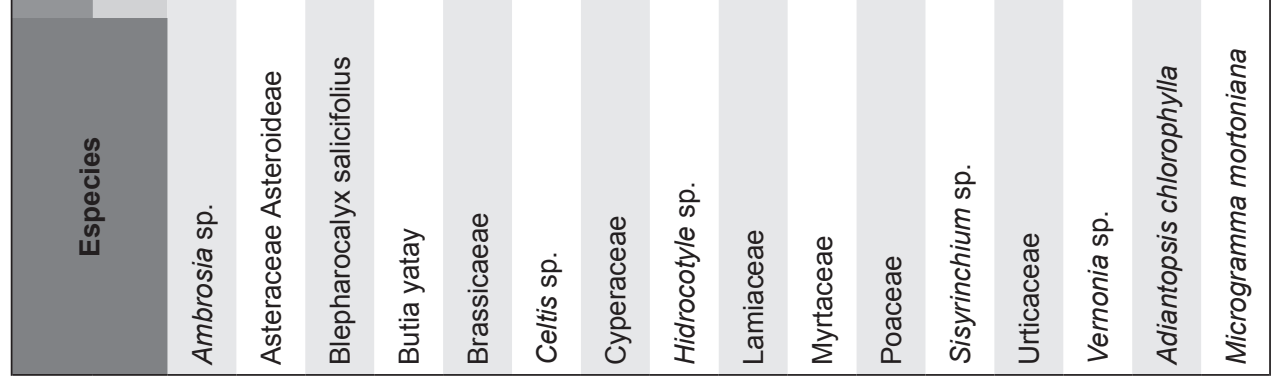


Bol. Soc. Argent. Bot. 52 (3) 2017

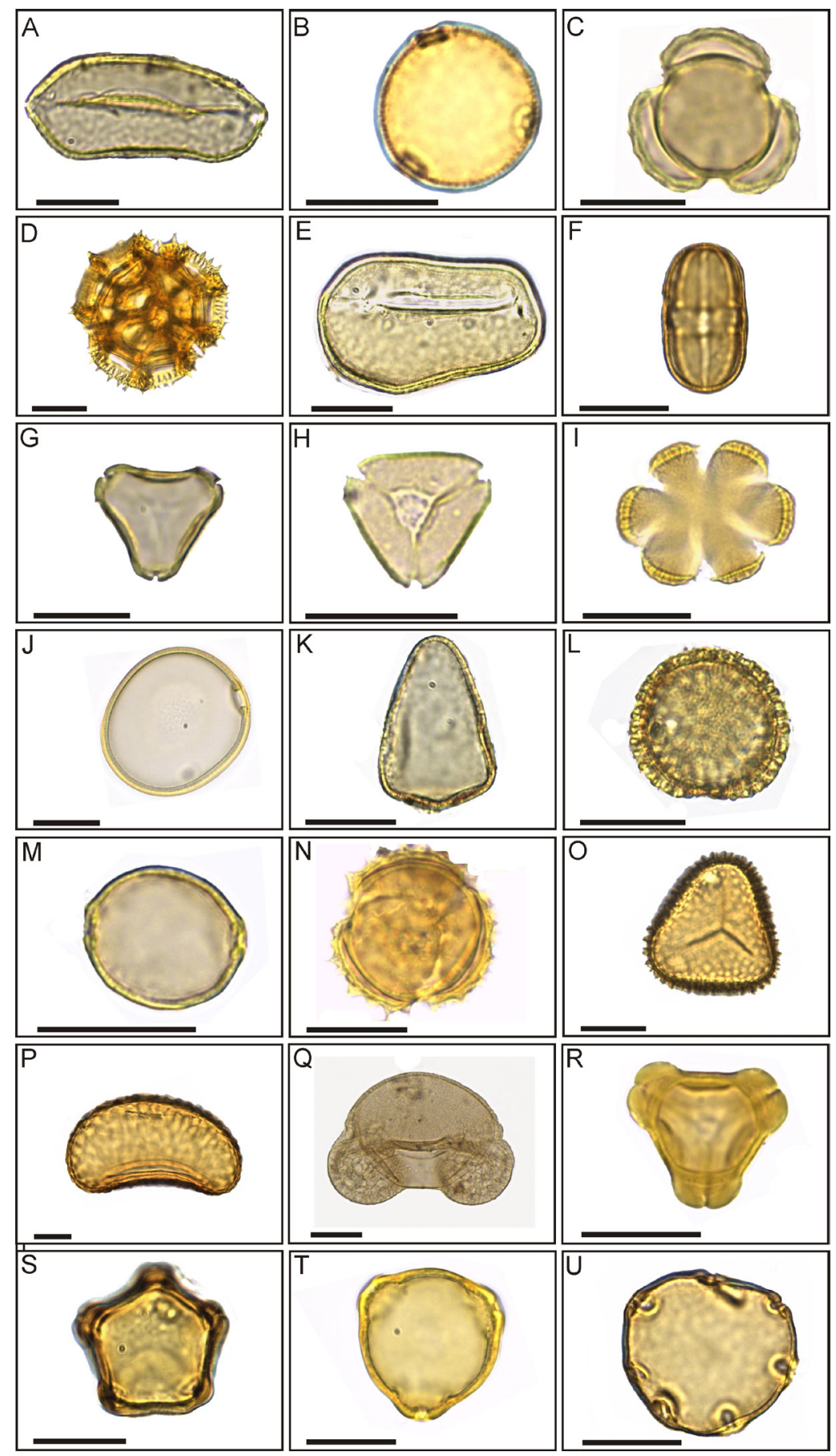

Fig. 4. Taxones considerados abundantes en el registro polínico, A- Butia yatay, B- Celtis sp., C- tipo Ambrosia sp., D- Vernonia sp., E- Sisyrinchium sp., F- Hidrocotyle sp., G- Myrtaceae, H- Blepharocalyx salicifolius, I- Lamiaceae, J- Poaceae, K- Cyperaceae, L- Brassicaceae, M- Urticaeae, N-AsteraeceaeAsteroideae, O- Adiantopsis chlorophylla, P- Microgramma mortoniana. Taxones exóticos hallados en las trampas Tauber, Q- Pinus sp., R- Eucalyptus sp, S- tipo Alnus, T- Casuarina cunninghamiana, U- Nothofagus sp. Escala: $20 \mu \mathrm{m}$. 


\section{N. E. Muñoz et al. - Análisis aeropalinológico del Parque Nacional El Palmar}

taxones. Los taxones dominantes fueron Mnesithea (=Rottboelia) selloana (Poaceae), Convolvulaceae y Asteraceae del género Vernonia sp., Porophyllum lanceolatum y Baccharis dracunculifolia. En la zona de la trampa Tauber 3 se reconocieron 29 familias de plantas, y su registro polínico representó el $54,8 \%$ de las familias, entre las que dominaron Butia yatay, Baccharis dracunculifolia, Adiantopsis chlorophylla, Allophylus edulis (Sapindaceae), Cyperus rotundus (Cyperacae), Myrtaceae, Poaceae y Rhynchosia senna (Fabaceae). Del total de plantas censadas el 54,95\% corresponden a plantas con polinización zoófila y el $31,19 \%$ a plantas con polinización anemófila.

Se buscó testear la relación entre la vegetación censada en cada uno de los sitios (CT1-CT3) y los muestreos polínicos aeropalinológicos (T1-T3) considerando valores de presencia ausencia (1-0) y el índice de similitud Jaccard.

Análisis 1: se utilizaron seis unidades (3 censos de vegetación y 3 muestreos polínicos bianuales) y 34 taxones incluyendo sólo aquéllos que se encuentran en al menos un censo y una trampa Tauber. Este análisis dio como resultado la siguiente relación (expresada en modo parentético): (((T2 T3) T1) CT1) (CT2 CT3)) (Figura 5.1).

Análisis 2: se utilizaron seis unidades (3 censos de vegetación y 3 muestreos polínicos bianuales) y 64 taxones, incluyendo los 16 taxones más abundantes en el muestreo aeropalinológico. Este análisis dio como resultado la siguiente relación: (((T1 T3) T2) ((CT2 CT3)) CT1) (Figura5.2).

Análisis 3: se utilizaron seis unidades (3 censos de vegetación y 3 muestreos polínicos bianuales) y los 16 taxones más abundantes en el muestreo polínico. Este análisis dio como resultado la siguiente relación: (((T1 T3) T2) (CT2 CT3) CT1) (Figura 5.3).

Como resultado general puede observarse que en los análisis se presenta un grupo constituido por la vegetación censada en los sitios CT2 y CT3 y otro grupo constituido por las muestras Tauber (T1, T2 y T3). En los análisis 1 y 2, las relaciones presentan baja similitud, en tanto que en el análisis 3 la similitud es mayor. Es de destacar lo que ocurre en el análisis 3 (16 taxones) en el cual las unidades T1 y T3 alcanzan la máxima similitud dado que presentan en común los 16 taxones seleccionados, en tanto que la unidad $\mathrm{T} 2$ registra la presencia de 15 de los 16 taxones.

\section{Taxones exóticos}

La vegetación regional está representada por tipos polínicos exóticos provenientes de la vegetación cercana al parque (Alnus, Casuarina) y de los alrededores del parque (Pinus sp., Eucalyptus sp.). Eucalyptus sp. presentó su mayor valor en Tauber 1, durante el primer año (7883,21 API), Alnus sp. y Casuarina sp. tuvieron su mayor valor durante el segundo año en la Tauber 3 (1492,35 y 19565,25 API, respectivamente); Pinus sp. se halló como más abundante en Tauber 3 con un valor de 7456,14 API durante e1 primer año y Nothofagus sp. en Tauber 2 con un valor de 356,50 API, durante el segundo año. Nothofagus sp. (extraregional) fue hallado en bajo número en los sitios 2 y 3 .

\section{Discusión}

Los resultados obtenidos del análisis del contenido palinológico (espectros polínicos) de los sitios de estudio (Figura 3.1), reflejan las características estructurales de la vegetación, definidas a través de atributos cualitativos y cuantitativos.

El Parque Nacional El Palmar se caracteriza por presentar ambientes con vegetación variada en la cual predominan un grupo de especies sobre otras en función del tipo de suelo y cobertura vegetal (canopeo, competencia). En este trabajo se consideraron cinco tipos de comunidad vegetal, Palmar denso, Arbustal con palmeras aisladas, Estepa psamófila, Pajonal y Bosque ripario (Batista et al., 2014). Las trampas Tauber se ubicaron respectivamente en un stand de estepa psamófila pajonal (trampa Tauber 1), en una estepa psamófila (trampa Tauber 2), y en un palmar denso (trampa Tauber 3). En términos de la clasificación de Batista et al. (2014), la trampa Tauber 1 se correspondería con la comunidad vegetal $\mathrm{F}$ (Estepa psamófila húmeda); la trampa Tauber 2 con la comunidad vegetal E (Estepas psamófilas); en tanto que la trampa Tauber 3 con la comunidad vegetal A (Palmar denso) (Figura 1).

Los diferentes factores que influyen en los cambios cuali-cuantitativos en el intervalo de los sitios estudiados (e.g. afinidad de polinización, procedencia de los granos de polen, efecto de canopía, variables meteorológicas) fueron considerados para caracterizar las comunidades de cada sitio. 
Bol. Soc. Argent. Bot. 52 (3) 2017

1

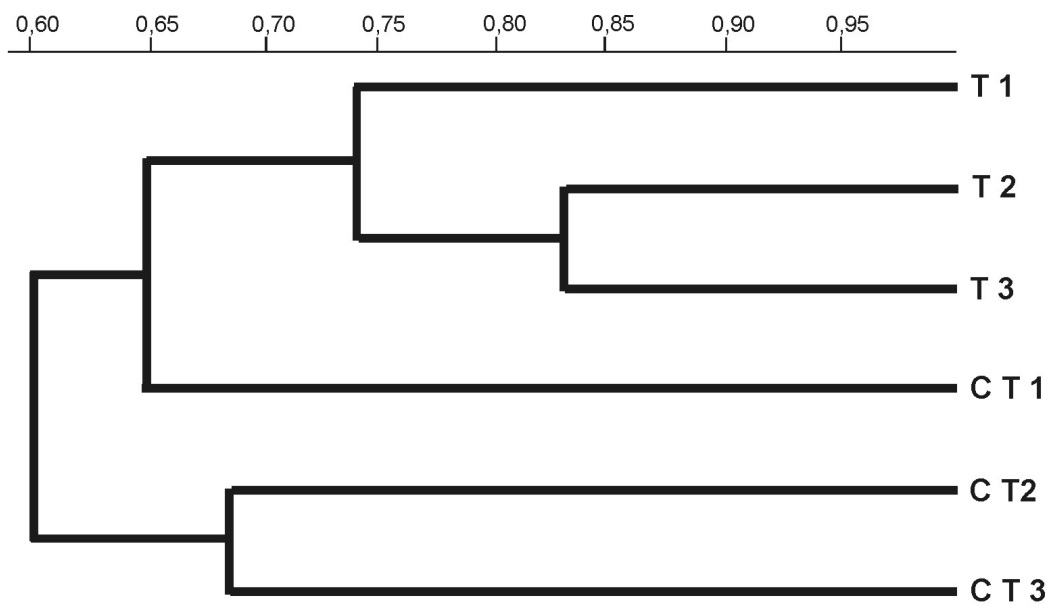

2

$\begin{array}{lllllllll}\perp^{0,32} & 0,40 & 0,48 & 0,56 & 0,64 & 0,72 & 0,80 & i_{i}^{i} & \end{array}$

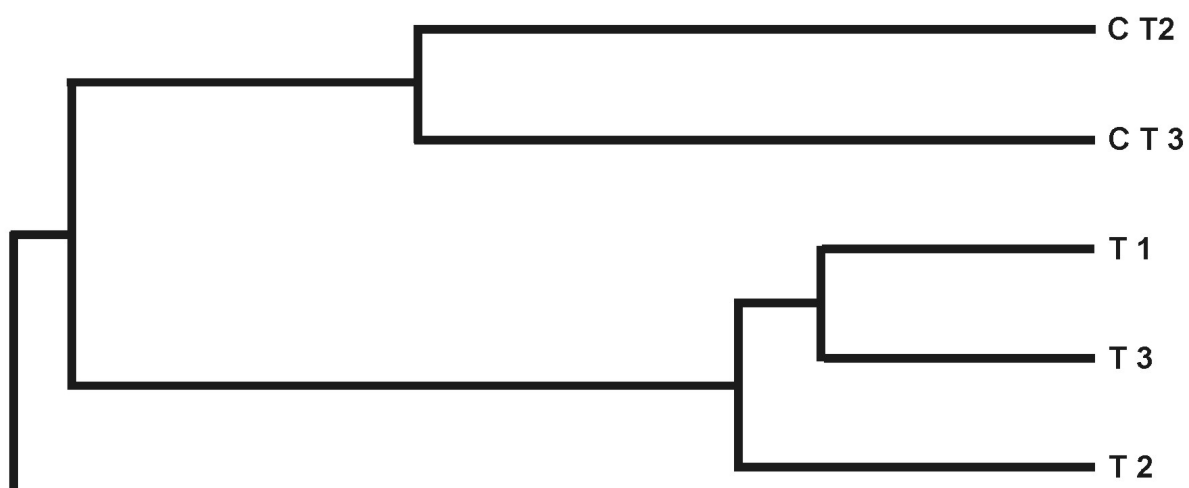

C T 1

3

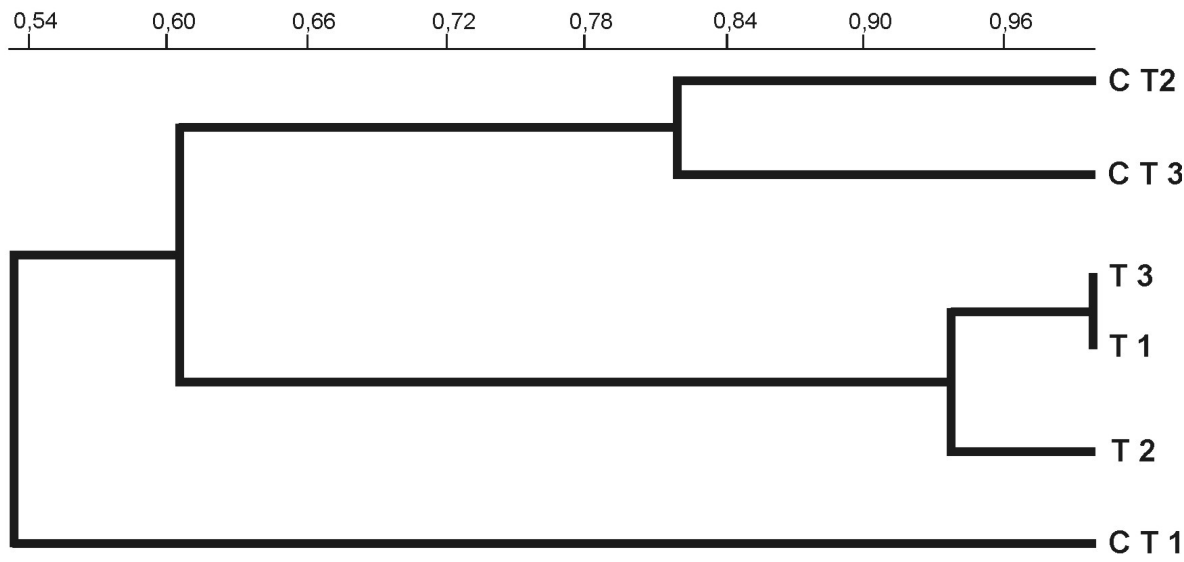

Fig. 5. Relación vegetación-polen. 1) utilizando 6 unidades y 34 taxones; 2) 6 unidades y 64 taxones; 3). 6 unidades y 16 taxones. Índice de similitud Jaccard. 


\section{N. E. Muñoz et al. - Análisis aeropalinológico del Parque Nacional El Palmar}

\section{Diversidad y abundancia en el registro polínico}

Las trampas Tauber 1 y Tauber 3 muestran una composición similar (i.e., Asteraeceae-Asteroideae, Myrtaceae, Blepharocalyx salicifolius, Poaceae), aunque con variaciones en los valores de API entre los dos años de muestreo. Sin embargo se diferencian en la mayor abundancia de Hidrocotyle sp, Ambrosia sp. y Brassicaceae en Tauber 1 y de Butia yatay, Adiantopsis chlorophylla y Microgramma mortoniana en Tauber 3. Tauber 2 se caracteriza por una alta abundancia de AsteraceaeAsteroideae, registrándose un aumento en el valor de API de Vernonia sp. (Asteraceae Cichoroideae) y Lamiaceae durante el segundo año (Tabla 3).

Asteraceae-Asteroideae y Poaceae son tipos polínicos multitaxones, en los cuales intervienen muchas especies de plantas que pueden aportar al espectro polínico, dependiendo de la región de estudio, y presentan un período de floración muy amplio, por lo cual pueden encontrarse granos de polen en la atmósfera prácticamente durante todo el año. Las Poaceae poseen polinización anemófila, por lo que tienen gran productividad de polen y dispersión. En el caso de Asteraceae-Asteroideae, muchas especies son anemófilas pero también hay especies de dispersión entomófila (e.g., Baccharis, Eupatorieae) (Tabla 1).

En el PNEP y de acuerdo a los censos realizados, la familia Myrtaceae está representada por Eugenia myrcianthes (=Hexachlamis edulis), Myrcianthes cysplatensis, Myrcia selloi y Psidium incanum. Las especies de Myrtaceae no se encontraron en los censos realizados en el área de las trampas Tauber 1 y Tauber 3, pero fueron halladas en zonas cercanas. Estas especies son entomófilas y en general sus granos de polen no se presentan de forma abundante en la atmósfera, excepto que las fuentes emisoras se encuentren próximas al sitio de muestreo polínico (Latorre, 1999; Latorre \& Caccavari, 2006; Nitiu, 2009). Probablemente debido a esto último el polen de Myrtaceae citado aparece como abundante en las trampas Tauber 1 y 3. Esta misma situación se registra en el tipo Blepharocalyx salicifolius, el cual resulta abundante en las trampas Tauber 1 y 3, aunque no fue hallada en los censos. La otra explicación puede estar vinculada con la presencia de insectos, que si bien se encontraron en baja frecuencia pueden haber influido en la sobrerrepresentación de los mencionados taxones.
Este rasgo será testeado a fin de clarificarlo.

Butia yatay posee polinización zoófila contando entre sus polinizadores abejas, escarabajos y murciélagos (Tomlinson, 1987; Lunazzi, 2009). Posee una alta producción de frutos y semillas, los cuales atraen pájaros frugívoros que dispersan sus semillas. Esta especie no florece todos los años y además tiene baja producción de polen (Mourrelle et al., 2016). Esto se refleja en el bajo valor de API en las Trampas durante todo el año, incluso en la zona de palmares (Tauber 3) con menores excepciones durante el período de agosto a diciembre (Tabla 2).

Adiantopsis chlorophylla es una especie de helecho terrestre de gran cobertura y alta frecuencia dentro de "chilcales" (Baccharis sp.), pastizales y palmares (Cottani \& Sabattini, 2006; Batista et al., 2014; di Pasquo et al., 2016). Esta especie produce una gran cantidad de esporas lo cual se constató en el registro polínico en los sitios de las trampas Tauber 1 y 3 , sin embargo en la trampa Tauber 2 es bajo (menos $1 \%$ ) reflejándose esta relación de cobertura en los censos.

Microgramma mortoniana es una especie de helecho epífito que crece sobre el tronco de palmeras, tal como fue hallada en los censos de vegetación y también se registra en los bosques en galería dentro del parque (di Pasquo et al., 2016). Esta distribución se ve reflejada en su alto valor API relativo durante los dos años de muestreo en la trampa Tauber 3.

Vernonia sp. resultó abundante tanto en los censos de vegetación realizados para el sitio de la trampa Tauber 2, como en sus recuentos polínicos.

Cyperaceae se encontró representado por varias especies como Carex sp., Cyperus reflexus, Cyperus rotundus y Bulbostylis capillaris en los censos de vegetación. Su abundancia en las muestras palinológicas de la trampa Tauber 1 coincide con lo hallado en los censos.

Sisyrinchium sp. aparece abundante en los recuentos polínicos de los dos años en la trampa Tauber 3, aunque se registró en baja frecuencia en los censos de vegetación.

Hidrocotyle sp. resultó abundante en los censos de vegetación y en los recuentos polínicos en la trampa Tauber 1 a pesar de poseer polinización entomófila, lo mismo ocurre con Celtis ehrenbergiana que no fue contabilizado en los censos pero fue observada en áreas circundantes a los sitios de las trampas 
Tauber 1 y 3. De igual forma este sesgo será testeado en un estudio próximo.

En los censos de vegetación no se hallaron ejemplares de Ambrosia sp. y Urticaceae pero al poseer polinización anemófila su presencia en las trampas Tauber puede sugerir que proviene de fuentes extra-locales o regionales (Tabla 2).

\section{Variaciones estacionales}

La mayoría de los tipos polínicos hallados en las trampas Tauber durante los dos años de muestreo corresponden a plantas presentes en el Parque Nacional El Palmar y particularmente a aquéllas registradas en las zonas donde están ubicadas las trampas.

El registro polínico estudiado muestra variaciones interanuales (Figura 4). Sin embargo, para los dos años se observa que la mayor acumulación polínica ocurre entre septiembre y febrero asociado al principal período de floración de la vegetación, y el mínimo entre junio y agosto junto con las más bajas temperaturas del año. Sin embargo en Tauber 3 se observa una distribución de picos estacionales más comparables entre el primer año y el segundo, en cambio en Tauber 1 se destaca un aumento significativo en el segundo año especialmente en los meses de septiembre y octubre en relación al primer año. Por su parte, Tauber 2 muestra también una variación importante principalmente en el segundo año siendo el mes de octubre el de mayor abundancia.

Cabe señalar que el mes de marzo de 2011 resultó ser el pico más importante de las tres Tauber, posiblemente atribuible a una floración profusa principalmente de las Asteraecae registrada además, en el censo de vegetación.

Por lo tanto, una explicación de la mayor variación interanual encontrada en las Tauber 1 y 2 podría deberse a que ambas se encuentran ubicadas en áreas abiertas donde la lluvia polínica puede ser afectada por el viento, mientras que el área donde se ubica la trampa Tauber 3 se distingue por presentar palmeras en una densidad elevada y junto con el estrato arbustivo producen una barrera contra el viento, cuyo efecto es aquí evidenciado por una mayor equidad en los valores anuales registrados.

Por otro lado, en Tauber 3 se registró un aumento de Microgramma mortoniana (asociada a Butia yatay) en el segundo año en concordancia con el incremento de Adiantopsis chlorophylla corroborado también por los censos de vegetación (Tabla 2), lo cual podría deberse a una relativa mayor temperatura y humedad (Figura 4).

\section{Comparación del registro polínico con la vegetación}

Como fue mencionado en el sitio Tauber 1 se reconocieron 31 familias de plantas y de ellas el registro aeropolínico representó $45,2 \%$ de las familias; en la zona de la trampa Tauber 2 se reconocieron 24 familias de plantas, el registro aeropolínico reflejó el 54,14\%, por último, en la zona de la trampa Tauber 3 se reconocieron 29 familias de plantas, el registro aeropolínico reflejó el 58,62 \% de las familias. El hecho de que en las trampas Tauber sólo se hayan identificado entre el 45 y el $58 \%$ de tipos polínicos que se corresponden a plantas censadas en dichos sitios podría deberse a una combinación de factores (e.g, composición, distribución y abundancia de los taxones en las comunidades vegetales, el tamaño, la producción y dispersión de polen, el tipo de polinización, la resistencia de los granos de polen a la degradación). Además, al considerar la diversidad de vegetación censada a nivel de género o especie (204 especies), comparativamente la diversidad hallada en las muestras palinológicas se reduce aún más dado que para algunas plantas sus granos de polen sólo pueden ser determinados a nivel de familia (Poaceae, Asteraceae, Myrtaceae, etc.) o resultaron granos no asignables a un taxón.

Pese a ciertas variaciones anuales en el predominio de los taxones la composición palinológica refleja y caracteriza la composición florística de cada sitio (Tabla 1, Tabla 2).

En el sitio Tauber 1 la vegetación está dominada por Poaceae, Cyperaceae y Juncaceae, en concordancia con el registro polínico donde los taxones dominantes fueron Poaceae y Cyperaceae. En tanto que Asteraceae, Myrtaceae y Blepharocalyx salicifolius son también abundantes pero no lo fueron en los censos. En particular, Blepharocalyx salicifolius no fue hallado en el área, sin embargo es un componente del bosque en galería que se encuentra rodeando el área (Figura 1). En el caso de las Juncaceae su polen presenta una pared delgada por lo que no resiste el proceso de acetólisis (Traverse, 2007). Por lo tanto, en términos polínicos este sitio se puede distinguir como una zona de ecotono pastizal-palmar y pajonal con bajos inundables a partir de la presencia dominante de 


\section{N. E. Muñoz et al. - Análisis aeropalinológico del Parque Nacional El Palmar}

Poaceae, Asteraceae, Cyperaceae y otros taxones como Myrtaceae y Butia yatay también presentes en la vegetación mixta del sitio.

El sitio Tauber 2 cuenta con taxones dominantes como las Asteraceae y Poaceae tanto en los censos como en el registro polínico. Sin embargo, en los censos Mnesithea selloana fue identificada como especie dominante, mientras que el polen se identificó a nivel de familia (Poaceae). Las Asteraceae Porphyllum lanceolatum y Baccharis dracunculifolia presentes en el sitio, fueron reconocidas a nivel de familia en el registro polínico mientras que Vernonia sp. pudo ser distinguida a nivel de género.

El sitio Tauber 3 que corresponde a un palmar denso, las plantas dominantes en los censos fueron principalmente las Asteraceae y coincidieron en abundancia con el registro polínico. La especie abundante en la vegetación fue Baccharis dracunculifolia que en los recuentos polínicos fue identificado a nivel de familia. En los censos Butia yatay fue muy abundante mientras que en los recuentos polínicos tuvo bajo valores pero con similares frecuencias anuales. Este bajo valor se explica por su característica de poseer una pared delgada, por lo cual puede verse afectado por el proceso de acetólisis (Traverse, 2007), sin descartar posiblemente su baja producción polínica debido a su polinización entomófila (Mourelle et al., 2016). Microgramma mortoniana es importante por cuanto se la asocia a Butia yatay, y su registro permite caracterizar especialmente áreas de palmares (Tabla 2), junto con la especie de helecho terrestre Adiantopsis chlorophylla, el cual al igual que las Poaceae, resultó abundante en los censos y en el recuento polínico.

Por otro lado, el mayor porcentaje de taxones encontrados en las trampas corresponde a plantas con polinización zoófila, contrario a lo que uno esperaría encontrar. Esto podría deberse a que la vegetación de este tipo se encuentra en la zona de las trampas Tauber. No obstante, otra razón podría vincularse con la presencia frecuente de insectos, sobre todo escarabajos, abejas, avispas y mariposas, los cuales podrían haber incorporado polen adherido a sus cuerpos. Como se mencionó más arriba, se realizará un estudio específico de este tema para evaluar el grado de influencia debido a la caída de insectos en las trampas, dado que las tapas utilizadas en este estudio no presentaron una malla para evitar su ingreso. Por lo tanto, la información obtenida hasta aquí podría tener este sesgo.

\section{Polen exótico}

La vegetación regional está representada principalmente por tipos polínicos exóticos presentes en la vegetación dentro del parque como Casuarina cunninghamiana que se encuentra plantada en la zona de la intendencia (Figura 1) y Alnus sp., que se reporta de establecimientos rurales cercanos. Además, la vegetación regional también está representada por taxones presentes en los alrededores del parque como Eucalyptus sp. y Pinus sp., los cuales forman parte de las numerosas plantaciones con fines madereros que se encuentran en zonas aledañas al PNEP. Estos tipos polínicos estuvieron representados a lo largo de los dos años de muestreo con variaciones interanuales.

Por otra parte, el polen extra-regional está representado por Nothofagus, taxón ampliamente distribuido en los bosques andinos patagónicos del suroeste de la Argentina (Faegri \& Iversen, 1989; Latorre et al., 2001; Trivi de Mandri et al., 2006), el cual aparece prácticamente en todos los muestreadores aerobiológicos de Argentina. Su transporte está asociado con el desplazamiento hacia el este por la circulación de vientos del oeste y la presencia de un sistema anticiclónico que traería el polen de Nothofagus hacia el norte como por ejemplo, fue registrado en las ciudades de Mar del Plata (Argentina) y Montevideo (Uruguay) (Tejera \& Beri, 2005; Gassmann \& Pérez, 2006). Este taxón fue encontrado en baja proporción entre agosto y diciembre en Tauber 2 y 3 concordando con su período de floración.

\section{Conclusiones}

En este trabajo se presenta el primer estudio de la relación polen-vegetación de los pastizales mesopotámicos del Este de la provincia de Entre Ríos. La agrupación de unidades aeropalinológicas en un cluster, y de unidades censo en otro podría explicarse en el hecho que varios de los taxones representados en cada una de estas unidades son exclusivos. Por lo tanto, para los análisis aquí presentados las asociaciones aeropalinológicas halladas en la trampas Tauber de un determinado sitio no representan de manera categórica a la vegetación presente en el sitio 
de muestreo. En particular, el polen hallado en las trampas Tauber, y que no se encuentra en los censos, puede provenir de taxones que se localizan próximos a las áreas de las trampas (no censados), o bien fue incorporado por los insectos caídos en las trampas. Los sitios Tauber presentan similares especies florísticas, aunque éstas no representan los grupos dominantes en sus comunidades vegetacionales respectivas. Una composición palinológica similar se halló en los sitios 1 y 3 . Sin embargo, el sitio 3 se diferenció de los otros dos por el registro de Butia yatay aunque en baja frecuencia y de los helechos Microgramma mortoniana y Adiantopsis chlorophylla especialmente durante el segundo año. El sitio 2 se caracterizó por la abundancia de Asteraeceae-Asteroideae, con un incremento en los valores de Vernonia (Asteraceae Cichoroidea) y Lamiaceae durante el segundo año. Se destaca la importancia de dar a conocer la diversidad florística desde el punto de vista palinológico pues contribuye a la caracterización del modelo análogo, el cual será utilizado en la reconstrucción de paleoambientes del Holoceno de la región de estudio.

\section{Agradecimientos}

Agradecemos muy especialmente al personal de Parque Nacional El Palmar por colaborar en el recambio de las trampas Tauber durante el período de realización del trabajo y hasta el presente. Además, se extienden los agradecimientos a la Administración de Parques Nacionales (APN) por autorizar el desarrollo del trabajo doctoral de N.M en el PNEP (Permiso 2016 No. NEA283 mv5), al CONICET por otorgarle la beca doctoral (2011-2017). Parte de esta investigación fue financiada con el subsidio PIP CONICET 593 dirigido por A.R. Prieto y con recursos propios de MDP. Al CICYTTP por autorizar el uso de los vehículos para los trabajos de campo y de las instalaciones y equipamientos del Laboratorio de Palinoestratigrafía y Paleobotánica (a cargo de MDP) donde se realizó el trabajo. Agradecemos a A.R. Prieto y D. Brandoni por su colaboración en los trabajos de campo.

\section{Biblografía}

BATISTA, W. B., A. G. ROLHAUSER, F. BIGANZOLI, S. E BURKART, L. GOVETO, A. MARANTA,
A. G. PIGNATARO, N. S. MORANDEIRA \& M. RABADÁN. 2014. Las Comunidades vegetales de la sabana del Parque Nacional El Palmar (Argentina). Darwiniana, nueva serie 2 (1): 5-38.

BORROMEI, A. M. \& M. QUATTROCCHIO. 1990. Dispersión del polen actual en el área de Bahía Blanca (Buenos Aires, Argentina). Anales Asoc. Palinol. Lengua Esp. 5: 39 - 52.

CICCERO, P. \& A. BALABUSIC. 1994. Plan de manejo preliminar del Parque Nacional El Palmar. Inédito. Buenos Aires: Administración de Parques Nacionales. Argentina. Inédito.

COTTANI, F. \& R SABATTINI. 2006. Manejo y control de arbustivas en un pastizal con altacarga animal en pastoreo rotativo. Rev. Cient. Agropec. 10 (2): 109-120.

CUADRADO, G. A. 1979. Calendario polínico preliminar para Corrientes (Argentina) y sus alrededores. Facena 3: 65-83.

DIMITRI, M. J. \& O. R. RIAL, (1955) La protección y conservación de la naturaleza en la Provincia de Entre Ríos. Natura, 1: 135-146.

DI PASQUO, M. \& L. J. SILVESTRI. 2014. Las colecciones de Palinología y Paleobotánica del Laboratorio de Palinoestratigrafía y Paleobotánica del Centro de Investigaciones Científicas y Transferencia de Tecnología a la Producción (CICyTTP), Entre Ríos, Argentina. Bol. Asoc. Latin. Paleobot. Palinol. 14: 39-47.

DI PASQUO, M., E. RODRÍGUEZ, N. NUÑEZ, N. E. MUÑOZ \& L. SILVESTRI. 2016. Esporas de helechos (Monilofita) y licofitas presentes en el Parque Nacional El Palmar, Provincia de Entre Ríos, Argentina. Bol. Soc. Argent. Bot. 51 (2): 269-298.

FAEGRI, K. \& J. IVERSEN. 1989. Textbook of Pollen Analysis. Chichester: Wiley. 328 pp.

FONTANA, S. L. 2003. Pollen deposition in coastal dunes, south Buenos Aires Province, Argentina. Rev. Palaeobot. Palynol. 126: 17-37.

FONTANA, S. L. 2004. Present and past coastal dune environments of South Buenos Aires Province, Argentina. Acta Universitatis Upsaliensis. Comprehensive Summaries of Uppsala Dissertations from the Faculty of Science and Technology, 940, Uppsala.

FONTANA, S. L., C. A. FERNÁNDEZ \& A. C. DEDOMENICI. 2001. Calendario polínico preliminar del área de Monte Hermoso, Buenos Aires, Argentina. En FOMBELLA BLANCO M. A., D. FERNÁNDEZ GONZÁLEZ, \& R. M. VALENCIA BARRERA (Eds.), Palinología: Diversidad y Aplicaciones. León, España: Universidad de León. pp. 227-233.

GASSMANN, M. I. \& C. F. PÉREZ. 2006. Trajectories associated to regional and extra-regional pollen 


\section{N. E. Muñoz et al. - Análisis aeropalinológico del Parque Nacional El Palmar}

transport in the southeast of Buenos Aires province, Mar del Plata (Argentina). Int J Biometeorol. 50: 280-291.

GOVETO, L. 2005. Ocurrencia histórica de fuegos en la sabana del Parque Nacional El Palmar: evidencias climáticas y florísticas. Tesis de maestría. Universidad de Buenos Aires. Facultad de Agronomía, Escuela para Graduados Alberto Soriano, p. 134.

HAMMER, Ø. D.A.T. HARPER\& P.D RYAN. 2001. PAST: Paleontological statistics software package for education and data analysis. Palaeontologia Electronica 4: 9 pp.

HEUSSER, C. J. 1971. Pollen and spores of Chile. Modern types of pteridophyta, Gymnospermae and Angiospermae. The University of Arizona Press, Tucson. pp 167.

HICKS, S. 1997. Pollen analogues and pollen influx values as a tool for interpreting the history of a settlement centre and its hinterland. PACT 52: 137-150.

HICKS, S. \& H. HYVÄRINEN. 1999. Pollen influx values measured in different sedimentary environments and their palaeoecological implications. Grana 38: 228242.

IRIONDO, M. \& D. KRÖHLING. 2008. Cambios ambientales en la cuenca del Uruguay (desde el Presente hasta dos millones de años atrás). Colección Ciencia y Técnica, Ediciones Universidad Nacional del Litoral, Santa Fe, pp. 360.

LATORRE, F. 1999. Difference between airbone pollen and flowering phenology of urban trees with referente to production, dispersal and interannual climate. Aerobiologia 15:131-141.

LATORRE, F., E. J. ROMERO \& M. V. MANCINI 2001. Representatividad de la vegetación en el espectro de polen atmosférico de la ciudad de Mar del Plata (Argentina). Asociación Paleontológica Argentina. Publicación Especial de Ameghiniana 8: 119-124. Buenos Aires, Argentina.

LATORRE, F. \& M. A. CACCAVARI. 2006. Depositación polínica anual en el Parque Nacional Pre-Delta (Entre Ríos, Argentina). Rev. Mus Argent. Ci. Nat. Bernardino Rivadavia 8: 195-200.

LATORRE, F., \& M. A. CACCAVARI. 2010. Diversidad polínica en el aire de Diamante (Entre Ríos, Argentina). Scient. Interfluv. (1-2): 7-17.

LUNAZZI, M. M. 2009. Estructura y dinámica poblacional de la palmera Butia yatay en la sabana del parque nacional el palmar: análisis en la escala de stand. Tesis de Magíster. Facultad de Agronomía. Escuela para Graduados Alberto Soriano. Universidad de Buenos Aires. pp 142.

MADANES, N. \& A. MILLONES. 2004. Estudio del polen aéreo y su relación con la vegetación en un agroecosistema. Darwiniana 42 (1-4): 51-62.
MAJAS, F. D. \& E. J. ROMERO. 1992. Aeropalynological research in the northeast of Buenos Aires Province, Argentina. Grana 31: 143-156.

MARATEO, G, H. POVEDANO \& J. ALONSO. 2009. Inventario de las aves del Parque Nacional El Palmar, Argentina. Cotinga 31: 47-60.

MARKGRAF, V. \& H.L. D’ANTONI. 1978. Pollen flora of Argentina. Modern spore and pollen types of Pteridophyta, Gymnospermae and Angiospermae. The University of Arizona Press, Tucson, AZ, pp 208.

MARTÍNEZ-CROVETTO, R., \& B. G. PICCININI, 1950. La vegetación de la República Argentina, 1. Los palmares de Butia yatay. Revista de Investigaciones Agricolas, 4: 153-242.

MOURRElle, D., P. GAIERO, G. SPERONI, C. MILAN, L. GUTIERREZ \& C. MAZZELLA. 2016. Comparative pollen morphology and viability among endangered species of Butia (Arecaceae) and its implications for species delimitation and conservation. Palynology 40 (2): 160-171.

MUELLER-DOMBOIS, D. \& H. ELLEMBERG. 1974. Aims and Methods of Vegetation Ecology. New York: Wiley \& Sons.

NAAB, O. A. 1999. Lluvia polínica actual en el Parque Nacional Lihuel-Calel, La Pampa, Argentina. Asociación Paleontológica Argentina. Publicación Especial de Ameghiniana 6: 85-89.

NITIU, D. 2009. Estudio del polen atmosférico y su relación con la vegetación local. La Plata, Argentina. Acta Botánica Malacitana 34: 189-199.

NUÑEZ OTAÑO, N., M. DI PASQUO \& N. MUÑOZ. 2015. Airborne fungal spore's identifications from three sites at the El Palmar National Park (Colón, Entre Ríos, Argentina): Their spatial and temporal variation. Aerobiologia 31: 537-547.

OVERBECK, G.E., S.E. MÜLLER, A. FIDELIS, J. PFADENHAUER, V.D. PILLAR, C.C. BLANCO, I.I. BOLDRINI, R. BOTH \& E.D. FORNECK. 2007. Brazil's neglected biome: The South Brazilian Campos. Perspectives in Plant Ecology, Evolution and Systematics, 9: 101-116.

PÉREZ, C. F., F. LATORRE, S. STUTZ \& S. PASTORINO. 2009. A two year report of pollen influx into Tauber traps in Mar Chiquita coastal lagoon, Buenos Aires, Argentina. Aerobiologia 25: 167-181.

PIRE S. M., M. ANZÓTEGUI L. \& G. A. CUADRADO. 1998. Flora polínica del nordeste argentino. Vol. 1 (EUDENE UNNE) Universidad Nacional del Nordeste, Argentina. pp 152.

PIRE S. M., M. ANZÓTEGUI L. \& G. A. CUADRADO. 2001. Flora polínica del nordeste argentino. Vol. 2 (EUDENE UNNE) Universidad Nacional del Nordeste, Argentina. pp. 172. 
PIRE S. M., M. ANZÓTEGUI L. \& G. A. CUADRADO. 2006. Flora polínica del nordeste argentino. Vol. 3 (EUDENE UNNE) Universidad Nacional del Nordeste, Argentina. pp 172.

ROLHAUSER A., F. BIGANZOLI, S. BURKART, L. GOVETO, A. MARANTA, G. PIGNATARO \& W. BATISTA. 2002. Comunidades vegetales de los palmares de Butia yatay: cambios asociados con la exclusión del ganado. XXIX Jornadas Argentinas de Botánica \& XV Reunión Anual de la Sociedad Botánica de Chile. p130.

ROLHAUSER, A. G. 2007. Regeneración de especies arbóreas nativas en los palmares de Butia yatay. Tesis de Maestría. Universidad de Buenos Aires. Facultad de Agronomía. Escuela para Graduados Alberto Soriano. pp 81.

ROLHAUSER, A. G., E. J CHANETON \& W. B. BATISTA. 2011. Influence of conspecific and heterospecific adults on riparian tree species establishment during encroachment of a humid palm savanna. Oecologia 167:141-48.

ROUBIK, D.W. \& J. E. MORENO. 1991. Pollen and Spores of Barro Colorado Island. Missouri Botanical Garden, Vol. 36, pp 270.

ROYO PALLARÉS, O., E.J. BERRETTA \& G.E. MARASCHIN. 2005. The South American Campos ecosystem. Grasslands of the World (eds J.Suttie, S.G. Reynolds, \& C. Batello) FAO. 171-219.

RUIZ SELMO, F. E., P. G. MINOTTI, A. SCOPEL \& M. PARIMBELLI. 2007. Análisis de la heterogeneidad fisonómico-funcional de la vegetación del Parque Nacional El Palmar y su relación con la invasión por leñosas exóticas. En: MARTIN (Ed.), Teledetección - Hacia un mejor entendimiento de la dinámica global y regional. 257-263 pp.
SÁNCHEZ, A. \& F. LATORRE. 2011. Floración de especies arbóreas urbanas y concentración de polen en el aire de Diamante (Entre Ríos, Argentina). Scientia Interfluvius 2(1): 07-19.

SORIANO,A., R.J.C.LEÓN, O.E. SALA, R.S. LAVADO, V.A. DEREGIBUS, O. CAHUEPÉ, A. SCAGLIA, C.A. VELAZQUEZ \& J.H. LEMKOFF 1991. Río de la Plata grasslands. En R.T. COUPLAND (Ed.), Natural Grasslands, Introduction and Western Hemisphere pp. 367-407. Elsevier. Amsterdam.

STOCKMAR, J. 1971. Tablets with spores used in absolute pollen analysis. Pollen et Spores 13: 615621.

TAUBER, H. 1974. A static non-overload pollen collector. New Phytol. 73: 359-369.

TEJERA, L. \& A. BERI. 2005. First volumetric airborne pollen sampling in Montevideo City, Uruguay. Aerobiología 21: 33-41.

TRAVERSE, A. 2007. Paleopalynology, 2nd. Edition. Springer, Dordrecht. The Netherlands. $813 \mathrm{pp}$.

TRIVI DE MANDRI, M. E., L. S. BURRY. \& H. L. D’ANTONI. 2006. Dispersión-depositación del polen actual en Tierra del Fuego, Argentina. Revista Mexicana de biodiversidad 77: 89-95.

ZULOAGA, F. O., O. MORRONE. \& M. J. BELGRANO (eds.). 2008. Catálogo de las plantas vasculares del Cono Sur. (Argentina, sur de Brasil, Chile, Paraguay y Uruguay). Monogr. Syst. Bot. Missouri Bot. Gard. 107: 1-3348. http://www2.darwin.edu.ar/Proyectos/ FloraArgentina.

Recibido el 7 de diciembre de 2016, aceptado el 9 de junio de 2017. 